\title{
A New Fractional Integration Operational Matrix of Chebyshev Wavelets in Fractional Delay Systems
}

\author{
Iman Malmir
}

Department of Mechanical Engineering, Buali Sina University, Hamedan, Iran; iman.malmir@outlook.com

Received: 30 June 2019; Accepted: 29 August 2019; Published: 3 September 2019

\begin{abstract}
Fractional integration operational matrix of Chebyshev wavelets based on the Riemann-Liouville fractional integral operator is derived directly from Chebyshev wavelets for the first time. The formulation is accurate and can be applied for fractional orders or an integer order. Using the fractional integration operational matrix, new Chebyshev wavelet methods for finding solutions of linear-quadratic optimal control problems and analysis of linear fractional time-delay systems are presented. Different numerical examples are solved to show the accuracy and applicability of the new Chebyshev wavelet methods.
\end{abstract}

Keywords: fractional integration operational matrix of Chebyshev wavelets; Chebyshev wavelets method; fractional time-delay optimal control; multifractional delay differential equation; quadratically constrained linear-quadratic optimal control; piecewise delay

\section{Introduction}

In some real-world problems, we deal with fractional calculus [1,2]. Fractional calculus has important engineering applications [3], for example, in the analysis of viscoelasticity structures $[4,5]$, in mechanics [6,7], control systems [8], and in partial differential equations [9] which they arise in many fields like Navier-Stokes equations that are of practical interest [10-12]. Fractional time-delay optimal control has been a topic of interest during recent years [13,14]. We know that governing state equations of some mechanical and control systems may result in second-order delay differential equations $[15,16]$. So, it is obvious that we may deal with the optimal control of multiorder fractional time-delay systems, in which a framework for the case when some fractional orders are greater than one will be needed. In previous works, some methods to handle optimization of delay systems have been presented, for example, in [17] by Bernoulli wavelets basis, in [18] by Legendre functions, in [19] by Boubaker functions, and in [20] by Chelyshkov wavelets. In these works however, it is assumed that there is a single fractional-order derivative in the state equation, control systems are unconstrained, the proposed solutions should be obtained from solving systems of algebraic equations, and also there is no discussion on the case when the fractional order is greater than one; it would therefore be helpful if general solutions for these situations could be found. Analysis of the different types of fractional differential equations [21] has been carried out in many texts by different methods, for example, [22-27]. In this paper, we study the optimal control and analysis of fractional delay systems by using Chebyshev wavelets [28] which have many advantages over Chebyshev polynomials such as compact support, providing accurate models of different types of delay systems, and a much wider range of capabilities. As we shall see later in this paper, general solutions of the optimal control and analysis of linear delay systems having fractional order state equations are presented. The fractional integration operational matrix of the conventional Chebyshev wavelets was presented in [29]. Additionally, in [30], another operational matrix of fractional order integration in the Riemann-Liouville sense was derived. However, these operational matrices were derived by employing block pulse and hat functions. It is desirable to have a general form of this matrix derived by employing Chebyshev 
wavelets, which is valid for all values of the order (integer and fractional). In this work, we obtain this matrix directly from Chebyshev wavelets via a new technique by which we can solve optimal control problems and delay differential equations having integer order and/or fractional order.

After stating some essential definitions in Section 2, by using the Riemann-Liouville fractional integral operator we introduce a new general formulation for the fractional integration operational of Chebyshev wavelets with scaling in Section 3, which is required for applying Chebyshev wavelet methods on fractional order systems. Then, in Section 4, we present new Chebyshev wavelet methods to the optimal control and analysis of fractional order systems having multiple delays and reverse time. The accuracy of the proposed Chebyshev wavelet methods by considering several examples are reported in Section 5. One can find from the results of the numerical examples that the proposed Chebyshev wavelet methods provide accurate results. Since the method for fractional linear-quadratic delay optimal control problems has the same idea to our previous method, it has some advantages (mentioned in [28] and [31]) over the existing method.

\section{Preliminaries}

Definition 1. Chebyshev Wavelets (CWs) and CWs Expansion

The arbitrary scaled Chebyshev wavelets (ASCWs) with five arguments are defined as [28]

$$
\psi_{n m}^{\xi}(t)=\psi(\xi, k, n, m, t)= \begin{cases}\sqrt{2 \xi^{k-1}} c_{m} T_{m}\left(2 \xi^{k-1} t-2 n+1\right), & t \in\left[\frac{n-1}{\xi^{k-1}}, \frac{n}{\xi^{k-1}}\right] \\ 0, & t \notin\left[\frac{n-1}{\xi^{k-1}}, \frac{n}{\xi^{k-1}}\right],\end{cases}
$$

where $\xi \in \mathbb{N}_{\geq 2}$ and is chosen from the problem $k \in \mathbb{N}_{\geq 2}, n=1,2, \ldots, \xi^{k-1}$ determines the location of a subinterval and refers to the subinterval number, $m=0,1, \ldots, M-1$ is the degree of $T_{m}$, the coefficient $c_{m}$ is $c_{0}=1 / \sqrt{\pi}, c_{m \neq 0}=\sqrt{2} / \sqrt{\pi}$, and $t \in[0,1]$ is the independent variable. The Chebyshev polynomials of the first kind $T_{m}(x)$ [32] are presented as

$$
T_{0}(x)=1, T_{m}(x)=m \sum_{j=0}^{m} \frac{(-2)^{j}(m+j-1) !}{(m-j) !(2 j) !}(1-t)^{j} \text { for } m>0 .
$$

We expand a function $f(t)$ in terms of Chebyshev wavelets over the interval $0 \leq t \leq 1$ as

$$
f(t)=\sum_{n=1}^{N} \sum_{m=0}^{\infty} f_{n m}^{\tau} \psi_{n m}^{\tau}(t)
$$

where $N$ is large enough. If we approximate $f(t)$, say, the $(M-1)$ th term in $\xi^{k-1}$ subintervals, then we have

$$
f(t) \cong \sum_{n=1}^{\xi^{k-1}} \sum_{m=0}^{M-1} f_{n m}^{\tau} \psi_{n m}^{\xi}(t)=\mathbf{f}_{c w} \mathbf{\Psi}_{\mathcal{\zeta}}(t)
$$

where $\mathbf{f}_{c w}$ and $\mathbf{\Psi}_{\xi}(t)$ are $1 \times \xi^{k-1} M$ and $\xi^{k-1} M \times 1$ vectors and

$$
\begin{gathered}
\mathbf{f}_{c w}:=\left[f_{10}^{\zeta}, \ldots, f_{1 M-1}^{\xi}, f_{20}^{\xi}, \ldots, f_{2 M-1}^{\zeta}, \ldots, f_{\xi^{k-1} 0^{\prime}}^{\xi}, \ldots, f_{\xi^{k-1} M-1}^{\xi}\right], \\
\mathbf{\Psi}_{\xi}(t):=\left[\psi_{10}^{\xi}(t), \ldots, \psi_{1 M-1}^{\xi}(t), \psi_{20}^{\xi}(t), \ldots, \psi_{2 M-1}^{\xi}(t), \ldots, \psi_{\xi^{k-1} 0}^{\xi}(t), \ldots, \psi_{\xi^{k-1} M-1}^{\xi}(t)\right]^{\top} .
\end{gathered}
$$

The constant coefficients $f_{n m}^{\tau}$ are obtained from

$$
f_{n m}^{\xi}=\frac{c_{m}}{\sqrt{2 \xi^{k-1}}} \int_{0}^{\pi} f\left(\frac{\cos \theta+2 n-1}{2 \xi^{k-1}}\right) \cos (m \theta) d \theta .
$$


Theorem 1. Let $f, f^{\prime}$, and $f^{\prime \prime}$ be piecewise continuous on the interval $[0,1]$, and $|f(t)|,\left|f^{\prime}(t)\right|,\left|f^{\prime \prime}(t)\right| \leq l$. Then, the accumulated error in $f,|e|=\left|f(t)-\mathbf{f}_{\mathcal{c} w} \mathbf{\Psi}_{\xi}(t)\right|$, for $M>2$ satisfies

$$
|e| \leq\left\{2+\frac{1}{N}+\frac{1}{\xi^{k-1}}+\frac{1}{4}\left(\frac{3}{2 N^{2}}+\frac{1}{\xi^{2 k-2}}\left\{\frac{3}{2}-\frac{2 M-1}{M(M-1)}\right\}\right)\right\} l .
$$

Proof. For the truncated CWs expansion given in (4), from (5) we obtain $\left|f_{n 0}^{\xi}\right|\left|\psi_{n 0}^{\xi}(t)\right| \leq l$, $\left|f_{n 1}^{\xi}\right|\left|\psi_{n 1}^{\xi}(t)\right| \leq l / \xi^{k-1}$ and $\left|f_{n m}^{\xi}\right|\left|\psi_{n m}^{\xi}(t)\right| \leq l /\left(2 \xi^{2 k-2}\left(m^{2}-1\right)\right)$. Similarly, for (3), we find $\left|f_{n^{\prime} 0}^{\zeta}\right|\left|\psi_{n^{\prime} 0}^{\zeta}(t)\right| \leq l,\left|f_{n^{\prime} 1}^{\xi}\right|\left|\psi_{n^{\prime} 1}^{\zeta}(t)\right| \leq l / N$ and $\left|f_{n^{\prime} m}^{\zeta}\right|\left|\psi_{n m}^{\xi}(t)\right| \leq l /\left(2 N^{2}\left(m^{2}-1\right)\right)$. We can write $|e|=\left|\sum_{n^{\prime}=1}^{N} \sum_{m=0}^{\infty} f_{n^{\prime} m}^{\zeta} \psi_{n^{\prime} m}^{\zeta}(t)-\sum_{n=1}^{\zeta^{k-1}} \sum_{m=0}^{M-1} f_{n m}^{\zeta} \psi_{n m}^{\xi}(t)\right|$. If $\xi^{k-1}=N$, we find $|e|=\left|\sum_{n=1}^{N} \sum_{m=M}^{\infty} f_{n m}^{\xi} \psi_{n m}^{\xi}(t)\right| \leq l(2 M-1) /\left(4 N^{2} M(M-1)\right)$. Otherwise, based on the value of $N$ and $\xi^{k-1}$ we consider two situations. 1. $\xi^{k-1}>N$; for $t \in\left[(n-1) / \xi^{k-1}, n / \xi^{k-1}\right]$, we have $|e|=\left|\sum_{m=0}^{\infty} f_{n^{\prime} m}^{\xi} \psi_{n^{\prime} m}^{\xi}(t)-\sum_{m=0}^{M-1} f_{n m}^{\xi} \psi_{n m}^{\zeta}(t)\right|$. We know $\left|\sum_{m=0}^{\infty} f_{n^{\prime} m}^{\xi} \psi_{n^{\prime} m}^{\xi}(t)\right| \leq l+l / N+$ $3 l /\left(8 N^{2}\right)$ and $\left|\sum_{m=0}^{M-1} f_{n^{\prime} m}^{\xi} \psi_{n^{\prime} m}^{\xi}(t)\right| \leq l+l / \xi^{k-1}+3 l /\left(8 \xi^{2 k-2}\right)-l(2 M-1) /\left(4 \xi^{2 k-2} M(M-1)\right)$. Hence, $|e|=\left|\sum_{m=0}^{\infty} f_{n^{\prime} m}^{\zeta} \psi_{n^{\prime} m}^{\zeta}(t)\right|+\left|\sum_{m=0}^{M-1} f_{n m}^{\zeta} \psi_{n m}^{\xi}(t)\right| \leq l+l / N+3 l /\left(8 N^{2}\right)+l+l / \xi^{k-1}+$ $3 l /\left(8 \xi^{2 k-2}\right)-l(2 M-1) /\left(4 \xi^{2 k-2} M(M-1)\right)$, which is equal to the given upper bound in the theorem. 2. $\xi^{k-1}<N$; in this situation, for $t \in\left[\left(n^{\prime}-1\right) / N, n^{\prime} / N\right]$, we can find a similar statement. Since $l(2 M-1) /\left(4 N^{2} M(M-1)\right) \leq\left\{2+\frac{1}{N}+\frac{1}{\xi^{k-1}}+\frac{1}{4}\left(\frac{3}{2 N^{2}}+\frac{1}{\xi^{2 k-2}}\left\{\frac{3}{2}-\frac{2 M-1}{M(M-1)}\right\}\right)\right\} l$, we have proved the theorem.

\section{Useful Properties of CWs}

We have the following properties of CWs which will be used for applying CW method:

1. $\int_{0}^{t} \boldsymbol{\Psi}_{\xi}(\varsigma) d \varsigma$ can be expressed in terms of $\boldsymbol{\Psi}_{\mathcal{\zeta}}(t)$ by the integration operational matrix of CWs denoted by $\mathbf{P}_{c w}$ as

$$
\int_{0}^{t} \Psi_{\xi}(\varsigma) d \varsigma \cong \mathbf{P}_{c w} \Psi_{\xi}(t)
$$

2. the product operational matrix of CWs for $\mathbf{f}_{c w}$ denoted by the symbol $\tilde{\mathbf{f}}_{c w}$ simplifies $\mathbf{f}_{c w} \boldsymbol{\Psi}_{\tilde{\zeta}}(t) \boldsymbol{\Psi}_{\xi}^{\top}(t)$ to

$$
\mathbf{f}_{c w} \mathbf{\Psi}_{\mathcal{\zeta}}(t) \mathbf{\Psi}_{\mathcal{\zeta}}^{\top}(t) \cong \mathbf{\Psi}_{\mathcal{\zeta}}^{\top}(t) \tilde{\mathbf{f}}_{c w} ;
$$

3. $\quad \Psi_{\mathcal{\zeta}}\left(t-h_{\iota}\right)$ and $\boldsymbol{\Psi}_{\mathcal{\xi}}(t-h(t))$, where $h_{\iota}$ is a time-delay and $h(t)$ is a piecewise delay, are expressed by the delay and the piecewise delay operational matrices of CWs denoted by the symbols, in turn, $\mathbf{D}_{l c w}$ and $\mathbf{D}_{c w}^{t}$, where

$$
\begin{gathered}
\boldsymbol{\Psi}_{\xi}\left(t-h_{\iota}\right)= \begin{cases}\mathbf{0}, & 0 \leq t<h_{\iota} \\
\mathbf{D}_{\iota c w} \mathbf{\Psi}_{\xi}(t), & h_{\iota} \leq t \leq 1,\end{cases} \\
\boldsymbol{\Psi}_{\xi}(t-h(t))= \begin{cases}\mathbf{0}, & 0 \leq t<h(t) \\
\mathbf{D}_{c w}^{t} \mathbf{\Psi}_{\xi}(t), & h(t) \leq t \leq 1 ;\end{cases}
\end{gathered}
$$

4. $\Psi_{\tilde{\zeta}}(1-t)$ is expressed by the inverse (reverse) time operational matrix of CWs denoted by $\Upsilon_{c w}$ as

$$
\mathbf{\Psi}_{\xi}(1-t)=\Upsilon_{c w} \mathbf{\Psi}_{\xi}(t) ;
$$

5. $\int_{0}^{1} \boldsymbol{\Psi}_{\xi}(t) \boldsymbol{\Psi}_{\mathcal{\zeta}}^{\top}(t) d t$ is obtained as the integration matrix of the product of two CWs vectors on $[0,1]$ denoted by $\boldsymbol{\Gamma}_{c w}$, that is,

$$
\int_{0}^{1} \Psi_{\mathcal{\zeta}}(t) \mathbf{\Psi}_{\mathcal{\zeta}}^{\top}(t) d t=\boldsymbol{\Gamma}_{c w}
$$

The procedure for constructing these matrices were explained in detail in [28].

Definition 2. Riemann-Liouville Fractional Integral and Caputo Fractional Derivative 
The Riemann-Liouville fractional integral operator of order $\alpha$ denoted by $\mathfrak{I}^{\alpha}$ is defined as

$$
\mathfrak{I}^{\alpha} f(t)= \begin{cases}\frac{1}{\Gamma(\alpha)} \int_{0}^{t}(t-\rho)^{\alpha-1} f(\rho) d \rho, & \alpha>0 \\ f(t), & \alpha=0,\end{cases}
$$

where $\Gamma$ is the gamma function. The Caputo fractional derivative of order $\alpha$ denoted by $D^{\alpha}$ is defined by

$$
D^{\alpha} f(t)=\mathfrak{I}^{\mathrm{n}-\alpha} D^{\mathrm{n}} f(t), \mathrm{n}-1<\alpha \leq \mathrm{n}, \mathrm{n} \in \mathbb{N} .
$$

$\mathfrak{I}^{\alpha} \rho^{m}$, where $\rho$ is the independent variable, possesses the property

$$
\mathfrak{I}^{\alpha} \rho^{m}=\frac{\Gamma(m+1)}{\Gamma(m+\alpha+1)} \rho^{m+\alpha} .
$$

Lemma 1. Let $f(t)$ be a continuous function. For $\alpha_{1}, \alpha_{2}>0$, we have

$$
\mathfrak{I}^{\alpha_{1}+\alpha_{2}} f(t)=\mathfrak{I}^{\alpha_{1}}\left[\mathfrak{I}^{\alpha_{2}} f(t)\right] .
$$

Proof. The proof can be found in [1].

The Caputo derivative arises in modeling of some physical system and has the remarkable property as

$$
\mathfrak{I}^{\alpha} D^{\alpha} f(t)=f(t)-\sum_{v=0}^{\mathrm{n}-1} f^{(v)}(0) \frac{t^{v}}{v !} .
$$

The Riemann-Liouville fractional integral and the Caputo fractional derivative operators denoted by $\mathfrak{L}$ are linear operators, that is, for functions $f(t)$ and $g(t)$ and scalars $a$ and $b$, we have

$$
\mathfrak{L}(a f(t)+b g(t))=a \mathfrak{L} f(t)+b \mathfrak{L} g(t) .
$$

\section{The Fractional Integration Operational Matrix of CWs}

In this section, we derive the fractional integration operational matrix of ASCWs which will be used in the next section.

Theorem 2. The fractional integration of ASCW vector $\boldsymbol{\Psi}_{\xi}(t)$ based on the Riemann-Liouville sense can be obtained directly from Chebyshev wavelets as

$$
\mathfrak{I}^{\alpha} \mathbf{\Psi}_{\xi}(t) \cong \mathbf{P}_{\mathcal{~} w}^{\alpha} \mathbf{\Psi}_{\mathcal{\zeta}}(t),
$$

where $\mathbf{P}_{c w}^{\alpha}$ is the $\xi^{k-1} M \times \xi^{k-1} M$ fractional integration operational matrix of $C W s$.

Proof. Using a procedure similar to that in [33], we see from the definition of this wavelet and (12) that when $t<n / \xi^{k-1}$, the integral is a function of the time over which the integrand is defined, so it should be expanded by the wavelets of the current subinterval $\left\{\psi_{n m}^{\xi}(t)\right\}$; when $t=n / \xi^{k-1}$, this definite integral should be expanded by ASCWs of all subsequent subintervals $\left\{\psi_{\eta m}^{\tau}(t)\right\}, \eta>n$. Assume that $n=1$. Hence, if $0 \leq t<1 / \xi^{k-1}$, we can write

$$
\mathfrak{I}^{\alpha} \psi_{1 m}^{\tau}(t)=z_{y}\left[\mathrm{y}_{10}^{\alpha m}, \mathrm{y}_{11}^{\alpha m}, \mathrm{y}_{12}^{\alpha m}, \ldots, \mathrm{y}_{1 M-1}^{\alpha m}\right]\left[\psi_{10}^{\tau}(t), \psi_{11}^{\xi}(t), \psi_{12}^{\tau}(t), \ldots, \psi_{1 M-1}^{\xi}(t)\right]^{\top},
$$

where $z_{y}$ is a constant and for $i=0,1,2, \ldots, M-1,\left\{\mathrm{y}_{1 i}^{\alpha m}\right\}$ are constants. We must find these parameters. From (1), we can write

$$
\mathfrak{I}^{\alpha} \psi_{1 m}^{\tau}(t)=\mathfrak{I}^{\alpha}\left\{\sqrt{2 \xi^{k-1}} \mathrm{c}_{m} T_{m}\left(2 \xi^{k-1} t-1\right)\right\} .
$$


In order to use (13), we must modify (2). Setting $-t \longrightarrow t$ yields $T_{m}(-t)=m \sum_{j=0}^{m} \frac{(-2)^{j}(m+j-1) !}{(m-j) !(2 j) !}(1+t)^{j}$. From $T_{m}(-t)=(-1)^{m} T_{m}(t)$, we find

$$
T_{m}(t)=(-1)^{m} m \sum_{j=0}^{m} \frac{(-2)^{j}(m+j-1) !}{(m-j) !(2 j) !}(1+t)^{j}, m>0 .
$$

Using (20), a more general formula for $T_{m}(t)$ is

$$
T_{m}(t)=(-1)^{m} \mathrm{a}_{m} \sum_{j=0}^{m} \frac{(-2)^{j}\left(\mathrm{a}_{m}+j-1\right) !}{(m-j) !(2 j) !}(1+t)^{j}, m \geq 0
$$

where $\mathrm{a}_{m}=\left\{\begin{array}{ll}1, & m=0 \\ m, & m \neq 0\end{array}\right.$. Now from (19), (13), and (21), we have

$$
\begin{aligned}
\mathfrak{I}^{\alpha} \psi_{1 m}^{\xi}(t) & =(-1)^{m} \sqrt{2 \xi^{k-1}} \mathrm{C}_{m} \mathrm{a}_{m} \mathfrak{I}^{\alpha} \sum_{j=0}^{m} \frac{(-2)^{j}\left(\mathrm{a}_{m}+j-1\right) !}{(m-j) !(2 j) !}\left(2 \xi^{k-1}\right)^{j} t^{j} \\
& =(-1)^{m} \sqrt{2 \xi^{k-1}} \mathrm{c}_{m} \mathrm{a}_{m} \sum_{j=0}^{m} \frac{(-2)^{j}\left(\mathrm{a}_{m}+j-1\right) !}{(m-j) !(2 j) !}\left(2 \xi^{k-1}\right)^{j} \frac{\Gamma(j+1)}{\Gamma(j+\alpha+1)} t^{j+\alpha} .
\end{aligned}
$$

By substituting the formula of $\mathfrak{I}^{\alpha} \psi_{1 m}^{\xi}(t)$ into (5), we can write

$$
\begin{aligned}
& (-1)^{m} \sqrt{2 \xi^{k-1}} \mathrm{c}_{m} \mathrm{a}_{m} \frac{\mathrm{c}_{i}}{\sqrt{2 \xi^{k-1}}} \int_{0}^{\pi} \sum_{j=0}^{m} \frac{(-2)^{j}\left(\mathrm{a}_{m}+j-1\right) !}{(m-j) !(2 j) !}\left(2 \xi^{k-1}\right)^{j} \frac{\Gamma(j+1)}{\Gamma(j+\alpha+1)}\left(\frac{\cos \theta+1}{2 \xi^{k-1}}\right)^{j+\alpha} \cos (i \theta) d \theta= \\
& \left(\frac{1}{2 \xi^{k-1}}\right)^{\alpha}(-1)^{m} \mathrm{a}_{m} \mathrm{c}_{m} \mathrm{c}_{i} \int_{0}^{\pi} \sum_{j=0}^{m} \frac{(-2)^{j}\left(\mathrm{a}_{m}+j-1\right) ! \Gamma(j+1)}{(m-j) !(2 j) ! \Gamma(j+\alpha+1)}(\cos \theta+1)^{j+\alpha} \cos (i \theta) d \theta .
\end{aligned}
$$

By $\boldsymbol{\varphi}_{n}^{\xi}(t):=\left[\psi_{n 0}^{\xi}(t), \psi_{n 1}^{\xi}(t), \psi_{n 2}^{\xi}(t), \ldots, \psi_{n M-1}^{\xi}(t)\right]^{\top}$, we see that $\mathfrak{I}^{\alpha} \boldsymbol{\Psi}_{\mathcal{\zeta}}(t)=\mathfrak{I}^{\alpha}\left[\boldsymbol{\varphi}_{1}^{\xi}(t), \mathbf{0}, \mathbf{0}, \cdots, \mathbf{0}\right]$. Hence, it follows from (18) for $0 \leq t<1 / \xi^{k-1}$ that

$$
\mathfrak{I}^{\alpha} \Psi_{\xi}(t)=\left(\frac{1}{2 \xi^{k-1}}\right)^{\alpha}\left[\begin{array}{ccccccc}
\mathbf{Y}_{10}^{\alpha} & \mathbf{0} & \mathbf{0} & \mathbf{0} & \cdots & \mathbf{0} & \mathbf{0} \\
\mathbf{Y}_{11}^{\alpha} & \mathbf{0} & \mathbf{0} & \mathbf{0} & \cdots & \mathbf{0} & \mathbf{0} \\
\vdots & \vdots & \vdots & \vdots & \ddots & \vdots & \vdots \\
\mathbf{Y}_{1 M-1}^{\alpha} & \mathbf{0} & \mathbf{0} & \mathbf{0} & \cdots & \mathbf{0} & \mathbf{0} \\
\hline \\
\left.\mathbf{0}_{\left(\xi^{k-1}\right.}-1\right) & M \times \xi^{k-1} M &
\end{array}\right] \boldsymbol{\Psi}_{\xi}(t)
$$

where

$$
\mathbf{Y}_{1 m}^{\alpha}=\left[\mathrm{y}_{10}^{\alpha m}, \mathrm{y}_{11}^{\alpha m}, \mathrm{y}_{12}^{\alpha m}, \ldots, \mathrm{y}_{1 M-1}^{\alpha m}\right]_{1 \times M}
$$

and

$$
\mathrm{y}_{1 i}^{\alpha m}=(-1)^{m} \mathrm{a}_{m} \mathrm{c}_{m} \mathrm{c}_{i} \int_{0}^{\pi} \sum_{j=0}^{m} \frac{(-2)^{j}\left(\mathrm{a}_{m}+j-1\right) ! \Gamma(j+1)}{(m-j) !(2 j) ! \Gamma(j+\alpha+1)}(\cos \theta+1)^{j+\alpha} \cos (i \theta) d \theta .
$$

When $t=1 / \xi^{k-1}$, we must have $\mathfrak{I}^{\alpha} \psi_{1 m}^{\xi}(t)=z_{j} \sum_{\eta=2}^{\xi^{k-1}}\left[\mathrm{j}_{\eta 0}^{\alpha m}, j_{\eta 1}^{\alpha m}, j_{\eta 2}^{\alpha m}, \ldots, j_{\eta M-1}^{\alpha m}\right] \boldsymbol{\varphi}_{\eta}^{\xi}(t)$, where $z_{j}$ and $\left\{\mathrm{j}_{\eta i}^{\alpha m}\right\}$ are constants. From (12),

$$
\mathfrak{I}^{\alpha} \psi_{1 m}^{\xi}(t)=\frac{1}{\Gamma(\alpha)} \int_{0}^{t}(t-\rho)^{\alpha-1} \sqrt{2 \xi^{k-1}} \mathrm{C}_{m} T_{m}\left(2 \xi^{k-1} \rho-1\right) d \rho .
$$


For $m=0$, we get $\mathfrak{I}^{\alpha} \psi_{10}^{\xi}(t)=\frac{1}{\Gamma(\alpha)} \int_{0}^{\frac{1}{\xi^{k-1}}}(t-\rho)^{\alpha-1} \sqrt{2 \xi^{k-1}} \mathrm{c}_{0} d \rho=\frac{\sqrt{2 \xi^{k-1}} \mathrm{c}_{0}}{\Gamma(\alpha+1)}\left(t^{\alpha}-\left(t-\frac{1}{\xi^{k-1}}\right)^{\alpha}\right)$. From this, the following statement about the higher degrees follows by induction

$$
\begin{aligned}
\mathfrak{I}^{\alpha} \psi_{1 m}^{\xi}(t)=(-1)^{m} \sqrt{2 \xi^{k-1}} \mathrm{c}_{m} \mathrm{a}_{m} \sum_{j=0}^{m} \frac{(-2)^{j}\left(\mathrm{a}_{m}+j-1\right) !}{(m-j) !(2 j) !}\left(2 \xi^{k-1}\right)^{j} \frac{\Gamma(j+1)}{\Gamma(j+\alpha+1)}\left(t^{j+\alpha}-\right. & \left.(-1)^{m-j}\left(t-\frac{1}{\xi^{k-1}}\right)^{j+\alpha}\right) .
\end{aligned}
$$

Substituting $t$, we find $\mathfrak{I}^{\alpha} \psi_{1 m}^{\xi}(t)=\left(\frac{1}{25^{k-1}}\right)^{\alpha} \sum_{\eta=2}^{\xi^{k-1}} \mathbf{J}_{\eta m}^{\alpha} \boldsymbol{\varphi}_{\eta}^{\xi}(t)$, where $\mathbf{J}_{\eta m}^{\alpha}=\left[\mathrm{j}_{\eta 0}^{\alpha m}, j_{\eta 1}^{\alpha m}, j_{\eta 2}^{\alpha m}, \ldots, j_{\eta M-1}^{\alpha m}\right]_{1 \times M}$. In the second interval $\eta=2$ and it is defined over $\left[1 / \xi^{k-1}, 2 / \xi^{k-1}\right]$. So, by $C_{m i}:=(-1)^{m} \mathrm{a}_{m} \mathrm{c}_{m} \mathrm{c}_{i}$ and setting $t=(\cos \theta+3) /\left(2 \xi^{k-1}\right)$, we have

$$
\mathrm{j}_{2 i}^{\alpha m}=C_{m i} \int_{0}^{\pi} \sum_{j=0}^{m} \frac{(-2)^{j}\left(\mathrm{a}_{m}+j-1\right) ! \Gamma(j+1)}{(m-j) !(2 j) ! \Gamma(j+\alpha+1)}\left\{(\cos \theta+3)^{j+\alpha}-(-1)^{m-j}(\cos \theta+1)^{j+\alpha}\right\} \cos (i \theta) d \theta .
$$

Additionally, in the last interval $\left[\left(\xi^{k-1}-1\right) / \xi^{k-1}, 1\right]$, where $\eta=\xi^{k-1}$, we find that

$$
\begin{aligned}
\mathbf{j}_{\xi^{k-1 i}}^{\alpha m}=C_{m i} \int_{0}^{\pi} \sum_{j=0}^{m} \frac{(-2)^{j}\left(\mathrm{a}_{m}+j-1\right) ! \Gamma(j+1)}{(m-j) !(2 j) ! \Gamma(j+\alpha+1)}\{ & \left(\cos \theta+2 \xi^{k-1}-1\right)^{j+\alpha} \\
& \left.-(-1)^{m-j}\left(\cos \theta+2 \xi^{k-1}-3\right)^{j+\alpha}\right\} \cos (i \theta) d \theta .
\end{aligned}
$$

Therefore, when $t=1 / \xi^{k-1}$

$$
\mathfrak{I}^{\alpha} \mathbf{\Psi}_{\xi}(t)=\left(\frac{1}{2 \xi^{k-1}}\right)^{\alpha}\left[\begin{array}{cccccccc}
\mathbf{0} & \cdots & \mathbf{0} & \mathbf{0} & \mathbf{J}_{20}^{\alpha} & \mathbf{J}_{30}^{\alpha} & \cdots & \mathbf{J}_{\xi_{k-1} 0}^{\alpha} \\
\mathbf{0} & \cdots & \mathbf{0} & \mathbf{0} & \mathbf{J}_{21}^{\alpha} & \mathbf{J}_{31}^{\alpha} & \cdots & \mathbf{J}_{\xi_{k-1} 1}^{\alpha} \\
\vdots & \ddots & \vdots & \vdots & \vdots & \vdots & \ddots & \vdots \\
\mathbf{0} & \cdots & \mathbf{0} & \mathbf{0} & \mathbf{J}_{2 M-1}^{\alpha} & \mathbf{J}_{3 M-1}^{\alpha} & \cdots & \mathbf{J}_{\xi_{k-1} M-1}^{\alpha} \\
\hline \multicolumn{1}{c}{\mathbf{0}_{\left(\xi^{k-1}-1\right) M \times \xi^{k-1} M}}
\end{array}\right] \Psi_{\xi}(t)
$$

where

$$
\begin{array}{r}
\mathbf{j}_{\eta i}^{\alpha m}=C_{m i} \int_{0}^{\pi} \sum_{j=0}^{m} \frac{(-2)^{j}\left(\mathrm{a}_{m}+j-1\right) ! \Gamma(j+1)}{(m-j) !(2 j) ! \Gamma(j+\alpha+1)}\left\{(\cos \theta+2 \eta-1)^{j+\alpha}-(-1)^{m-j}(\cos \theta+2 \eta-3)^{j+\alpha}\right\} \\
\cos (i \theta) d \theta .
\end{array}
$$

In general, if $(n-1) / \xi^{k-1} \leq t(n)<n / \xi^{k-1}$, where $t(n)=t+(n-1) / \xi^{k-1}$, we see

$$
\mathfrak{I}^{\alpha} \psi_{n m}^{\xi}(t(n))=\frac{1}{\Gamma(\alpha)} \int_{\frac{n-1}{\xi^{k-1}}}^{t(n)}(t(n)-\rho)^{\alpha-1} \sqrt{2 \xi^{k-1}} \mathrm{c}_{m} T_{m}\left(2 \xi^{k-1} \rho-2 n+1\right) d \rho .
$$

Substituting $\varepsilon=\rho-(n-1) / \xi^{k-1}$, gives us

$$
\mathfrak{I}^{\alpha} \psi_{n m}^{\xi_{m}}(t(n))=(-1)^{m} \sqrt{2 \xi^{k-1}} \mathrm{c}_{m} \mathrm{a}_{m} \sum_{j=0}^{m} \frac{(-2)^{j}\left(\mathrm{a}_{m}+j-1\right) !}{(m-j) !(2 j) !}\left(2 \xi^{k-1}\right)^{j} \frac{\Gamma(j+1)}{\Gamma(j+\alpha+1)}\left(t(n)-\frac{n-1}{\xi^{k-1}}\right)^{j+\alpha} .
$$

In this subinterval we must set $t(n)=(\cos \theta+2 n-1) /\left(2 \xi^{k-1}\right)$ to exanpd $\mathfrak{I}^{\alpha} \psi_{n m}^{\xi}(t(n))$ by the wavelets of the current subinterval. This gives $\left(2 \xi^{k-1}\right)^{j}\left(t(n)-(n-1) / \xi^{k-1}\right)^{j+\alpha}=\left(1 /\left(2 \xi^{k-1}\right)^{\alpha}\right)(\cos \theta+1)^{j+\alpha}$, 
so $\mathfrak{I}^{\alpha} \psi_{n m}^{\xi}(t(n))$ has the same coefficient as $\mathfrak{I}^{\alpha} \psi_{1 m}^{\xi}(t)$ in terms of the CWs vector, and for $t(n)<n / \xi^{k-1}$ we have

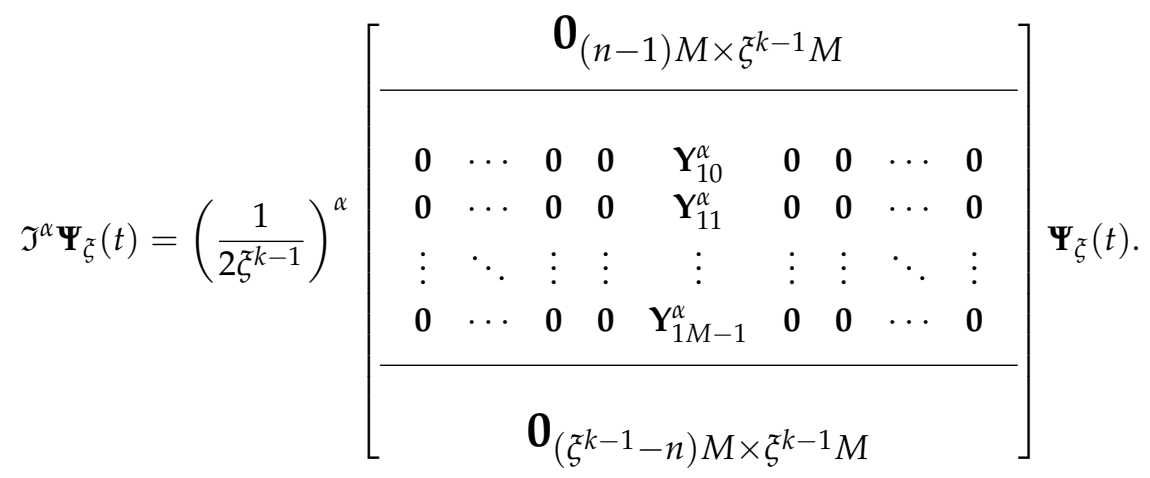

When $t(n)=n / \xi^{k-1}$, we find $\mathfrak{I}^{\alpha} \psi_{n 0}^{\xi}(t(n))=\frac{1}{\Gamma(\alpha)} \int_{\frac{n-1}{\xi^{k-1}}}^{\frac{n}{\xi^{k-1}}}(t(n)-\rho)^{\alpha-1} \sqrt{2 \xi^{k-1}} \mathrm{C}_{0} d \rho=\frac{\sqrt{2 \xi^{k-1}} c_{0}}{\Gamma(\alpha+1)}((t(n)-$ $\left.\left.\frac{n-1}{\xi^{k-1}}\right)^{\alpha}-\left(t(n)-\frac{n}{\xi^{k-1}}\right)^{\alpha}\right)$. Also we see that $\Im^{\alpha} \psi_{n 1}^{\xi}(t(n))=\frac{1}{\Gamma(\alpha)} \int_{\frac{\xi^{k-1}}{\xi^{k-1}}}^{\frac{n}{\xi^{k-1}}}(t(n)-\rho)^{\alpha-1} \sqrt{2 \xi^{k-1}} \mathrm{c}_{1}\left(2 \xi^{k-1} \rho-\right.$ $2 n+1) d \rho=-\frac{\sqrt{2 \xi^{k-1}} c_{1}}{\Gamma(\alpha+1)}\left(\left(t(n)-\frac{n-1}{\xi^{k-1}}\right)^{\alpha}+\left(t(n)-\frac{n}{\xi^{k-1}}\right)^{\alpha}\right)+\frac{\sqrt{2 \xi^{k-1}} c_{1}}{\Gamma(\alpha+2)}\left(2 \xi^{k-1}\right)\left(\left(t(n)-\frac{n-1}{\xi^{k-1}}\right)^{1+\alpha}-(t(n)-\right.$ $\left.\left.\frac{n}{\xi^{k-1}}\right)^{1+\alpha}\right)$. By induction, we get the following statement for the higher degrees

$$
\begin{aligned}
\mathfrak{I}^{\alpha} \psi_{n m}^{\xi}(t(n))=(-1)^{m} \sqrt{2 \xi^{k-1}} \mathbf{c}_{m} \mathbf{a}_{m} \sum_{j=0}^{m} \frac{(-2)^{j}\left(\mathbf{a}_{m}+j-1\right) !}{(m-j) !(2 j) !}\left(2 \xi^{k-1}\right)^{j} \frac{\Gamma(j+1)}{\Gamma(j+\alpha+1)} \\
\left(\left(t(n)-\frac{n-1}{\xi^{k-1}}\right)^{j+\alpha}-(-1)^{m-j}\left(t(n)-\frac{n}{\xi^{k-1}}\right)^{j+\alpha}\right) .
\end{aligned}
$$

$\mathfrak{I}^{\alpha} \psi_{n m}^{\tilde{\zeta}}(t(n))$ must be expanded in its next subintervals, that is, $(n+1)-\mathrm{th},(n+2)-$ th, $\ldots,\left(\xi^{k-1}\right)-$ th subintervals, so that $\mathfrak{I}^{\alpha} \psi_{n m}^{\tau}(t(n))=\sum_{\eta=n+1}^{\xi^{k-1}} \mathbf{J}_{\eta m}^{\alpha} \boldsymbol{\varphi}_{\eta}^{\xi}(t(\eta))$. Hence, when $t(n)=n / \xi^{k-1}$, we obtain

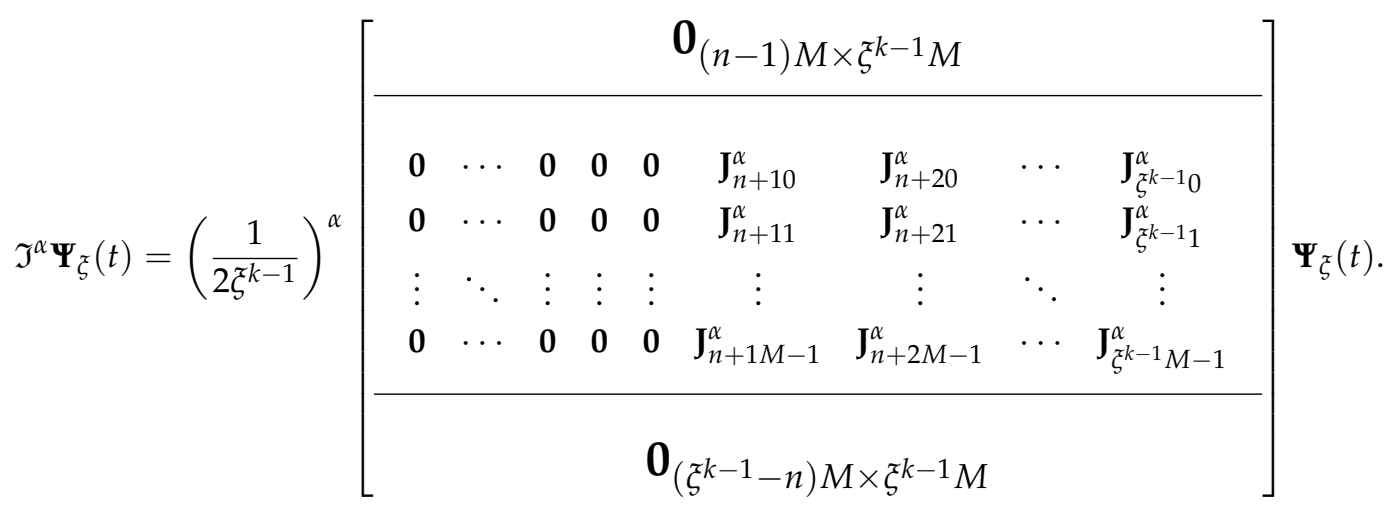

For $\eta=n+1$ by $t(n)=(\cos \theta+2 \eta-1) /\left(2 \xi^{k-1}\right)$, we find

$$
\mathrm{j}_{\eta i}^{\alpha m}=C_{m i} \int_{0}^{\pi} \sum_{j=0}^{m} \frac{(-2)^{j}\left(\mathrm{a}_{m}+j-1\right) ! \Gamma(j+1)}{(m-j) !(2 j) ! \Gamma(j+\alpha+1)}\left\{(\cos \theta+3)^{j+\alpha}-(-1)^{m-j}(\cos \theta+1)^{j+\alpha}\right\} \cos (i \theta) d \theta .
$$

In general, $\mathrm{j}_{\eta i}^{\alpha m}$ is obtained from

$$
\begin{array}{r}
C_{m i} \int_{0}^{\pi} \sum_{j=0}^{m} \frac{(-2)^{j}\left(\mathrm{a}_{m}+j-1\right) ! \Gamma(j+1)}{(m-j) !(2 j) ! \Gamma(j+\alpha+1)}\left\{(\cos \theta+2 \eta-2 n+1)^{j+\alpha}-(-1)^{m-j}(\cos \theta+2 \eta-2 n-1)^{j+\alpha}\right\} \\
\cos (i \theta) d \theta .
\end{array}
$$


We find that $\left.\mathbf{J}_{n+1 m}^{\alpha}\right|_{\eta=n+1}=\left.\mathbf{J}_{2 m}^{\alpha}\right|_{\eta=2},\left.\mathbf{J}_{n+2 m}^{\alpha}\right|_{\eta=n+1}=\left.\mathbf{J}_{3 m}^{\alpha}\right|_{\eta=3}, \ldots$, and $\left.\mathbf{J}_{\xi^{k-1} m}^{\alpha}\right|_{\eta=n+1}=\left.\mathbf{J}_{\xi^{k-1}-(n-1) m}^{\alpha}\right|_{\eta=2}$, where $\left.\mathbf{J}_{n+1 m}^{\alpha}\right|_{\eta=n+1}$ indicates the computed $\mathbf{J}_{n+1 m}^{\alpha}$ for $(n+1)$-th subinterval. Taking (22)-(25) together and by

$$
\mathbf{Y}^{\alpha}:=\left[\begin{array}{c}
\mathbf{Y}_{10}^{\alpha} \\
\mathbf{Y}_{11}^{\alpha} \\
\vdots \\
\mathbf{Y}_{1 M-1}^{\alpha}
\end{array}\right], \mathbf{J}_{\eta-1}^{\alpha}:=\left[\begin{array}{c}
\mathbf{J}_{\eta 0}^{\alpha} \\
\mathbf{J}_{\eta 1}^{\alpha} \\
\vdots \\
\mathbf{J}_{\eta M-1}^{\alpha}
\end{array}\right]
$$

we have $\mathfrak{I}^{\alpha} \mathbf{\Psi}_{\xi}(t) \cong \mathbf{P}_{c w}^{\alpha} \mathbf{\Psi}_{\xi}(t)$, where

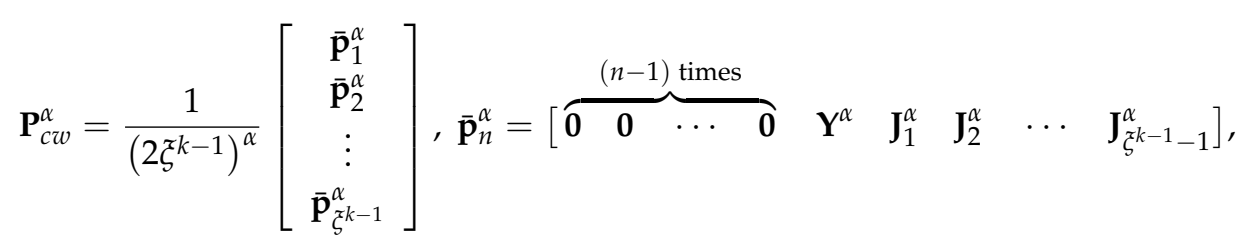

in which $\mathbf{0}$ is a zero matrix of order $M$ and the proof is complete.

Corollary 1. Setting $\eta-1 \longrightarrow \eta$, the fractional integration operational matrix of $C W s \mathbf{P}_{c w}^{\alpha}$ obtained from

$$
\mathbf{P}_{c w}^{\alpha}=\frac{1}{\left(2 \xi^{k-1}\right)^{\alpha}}\left[\begin{array}{cccccc}
\mathbf{Y}^{\alpha} & \mathbf{J}_{1}^{\alpha} & \mathbf{J}_{2}^{\alpha} & \mathbf{J}_{3}^{\alpha} & \cdots & \mathbf{J}_{\xi^{k-1}-1}^{\alpha} \\
\mathbf{0} & \mathbf{Y}^{\alpha} & \mathbf{J}_{1}^{\alpha} & \mathbf{J}_{2}^{\alpha} & \cdots & \mathbf{J}_{\xi^{k-1}-2}^{\alpha} \\
\vdots & \vdots & \vdots & \vdots & \ddots & \vdots \\
\mathbf{0} & \mathbf{0} & \mathbf{0} & \mathbf{0} & \cdots & \mathbf{Y}^{\alpha}
\end{array}\right]
$$

where

$$
\begin{gathered}
\mathbf{Y}^{\alpha}=\left[\begin{array}{ccccc}
\mathrm{y}_{10}^{\alpha 0} & \mathrm{y}_{11}^{\alpha 0} & \mathrm{y}_{12}^{\alpha 0} & \ldots & \mathrm{y}_{1 M-1}^{\alpha 0} \\
\mathrm{y}_{10}^{\alpha 1} & \mathrm{y}_{11}^{\alpha 1} & \mathrm{y}_{12}^{\alpha 1} & \ldots & \mathrm{y}_{1 M-1}^{\alpha 1} \\
\vdots & \vdots & \vdots & \ddots & \vdots \\
\mathrm{y}_{10}^{\alpha M-1} & \mathrm{y}_{11}^{\alpha M-1} & \mathrm{y}_{12}^{\alpha M-1} & \ldots & \mathrm{y}_{1 M-1}^{\alpha M-1}
\end{array}\right], \mathbf{J}_{\eta}^{\alpha}=\left[\begin{array}{ccccc}
\mathrm{j}_{\eta 0}^{\alpha 0} & \mathrm{j}_{\eta 1}^{\alpha 0} & \mathrm{j}_{\eta 2}^{\alpha 0} & \ldots & \mathrm{j}_{\eta M-1}^{\alpha 0} \\
\mathrm{j}_{\eta 0}^{\alpha 1} & \mathrm{j}_{\eta 1}^{\alpha 1} & \mathrm{j}_{\eta 2}^{\alpha 1} & \ldots & \mathrm{j}_{\eta M-1}^{\alpha 1} \\
\vdots & \vdots & \vdots & \ddots & \vdots \\
\mathrm{j}_{\eta 0}^{\alpha M-1} & \mathrm{j}_{\eta}^{\alpha M-1} & \mathrm{j}_{\eta 2}^{\alpha M-1} & \ldots & \mathrm{j}_{\eta M-1}^{\alpha M-1}
\end{array}\right], \\
\mathrm{y}_{1 i}^{\alpha m}=(-1)^{m} \mathrm{a}_{m} \mathrm{c}_{m} \mathrm{c}_{i} \int_{0}^{\pi} \sum_{j=0}^{m} \frac{(-2)^{j}\left(a_{m}+j-1\right) ! \Gamma(j+1)}{(m-j) !(2 j) ! \Gamma(j+\alpha+1)}(\cos \theta+1)^{j+\alpha} \cos (i \theta) d \theta, \\
\mathrm{j}_{\eta i}^{\alpha m}=(-1)^{m} \mathrm{a}_{m} \mathrm{c}_{m} \mathrm{c}_{i} \int_{0}^{\pi} \sum_{j=0}^{m} \frac{(-2)^{j}\left(a_{m}+j-1\right) ! \Gamma(j+1)}{(m-j) !(2 j) ! \Gamma(j+\alpha+1)}\left\{(\cos \theta+2 \eta+1)^{j+\alpha}-\right. \\
\left.(-1)^{m-j}(\cos \theta+2 \eta-1)^{j+\alpha}\right\} \cos (i \theta) d \theta .
\end{gathered}
$$

Corollary 2. The integration operational matrix of $C W s \mathbf{P}_{c w}$ in (6) is a particular case of the fractional integration operational matrix when $\alpha=1$, and the presented formulation can be used for this case.

Corollary 3. When we set $\xi=2$, the fractional integration operational matrix of $C W$ s with scaling $\mathbf{P}_{c w}^{\alpha}$ can be applied on the conventional Chebyshev wavelets.

Thus, the fractional integration operational matrix of CWs is obtained directly from Chebyshev scaling functions as claimed. In (28) and (29), we have some definite integrals which can be calculated by many available numerical methods. We will present some tests for the accuracy of the fractional integration operational matrix in illustrative examples. 


\section{Chebyshev Wavelet Methods for Fractional Delay Systems}

We present CW methods to the optimal control and analysis of fractional order delay systems in this section.

\subsection{Optimal Control of Linear-Quadratic Fractional Time-Delay Systems}

Consider a linear fractional time-delay system described by

$$
\begin{aligned}
& D^{\alpha} \mathbf{x}(t)=\mathbf{A}(t) \mathbf{x}(t)+\mathbf{B}(t) \mathbf{u}(t)+\sum_{\mu=1}^{a} \mathbf{E}_{\mu}(t) \mathbf{x}\left(t-h_{\mu}\right)+\sum_{v=1}^{b} \mathbf{F}_{v}(t) \mathbf{u}\left(t-h_{v}\right)+\mathbf{G}(t) \mathbf{x}(1-t)+\mathbf{d}(t), \\
& \mathbf{x}^{(i)}(0)=\mathbf{x}_{i}, \begin{cases}\mathbf{x}(t)=\theta(t) & -h_{x} \leq t<0 \\
\mathbf{u}(t)=\zeta(t) & -h_{u} \leq t \leq 0,\end{cases}
\end{aligned}
$$

where $0<\alpha \leq 2, t \in[0,1], \mathbf{x}(t)$ and $\mathbf{u}(t)$ are $q$ th state and $r$ th control vectors, $\mathbf{A}(t), \mathbf{B}(t), \mathbf{E}_{\mu}(t), \mathbf{F}_{v}(t)$, and $\mathbf{G}(t)$ are matrices of appropriate dimensions, $\mathbf{d}(t)$ as a $q$ th vector represents disturbances, $i=0,1$ and $\mathbf{x}^{(i)}$ denotes $\frac{d^{i} \mathbf{x}}{d t^{i}}, h_{\mu}$ and $h_{\nu}$ are delays, $\theta(t)$ and $\zeta(t)$ are, respectively, $q$ th and $r$ th specified initial vector functions, $h_{x}=\max \left\{\left\{h_{\mu}\right\}\right\}$ and $h_{u}=\max \left\{\left\{h_{\nu}\right\}\right\}$. The fractional time-delay system is to be controlled to minimize the quadratic performance index

$$
J=\frac{1}{2} \mathbf{x}^{\top}(1) \mathbf{T} \mathbf{x}(1)+\frac{1}{2} \int_{0}^{1}\left\{\mathbf{x}^{\top}(t) \mathbf{Q}(t) \mathbf{x}(t)+\mathbf{u}^{\top}(t) \mathbf{R}(t) \mathbf{u}(t)\right\} d t,
$$

where $\mathbf{T}$ and $\mathbf{Q}(t)$ are positive semidefinite matrices and $\mathbf{R}(t)$ is a positive definite matrix.

Depending on the value of $\alpha$, we consider two cases as A and B to solve the problem.

A. $0<\alpha \leq 1$

We take $h_{\mu}=\frac{\epsilon_{\mu}}{\omega_{\mu}}$ and $h_{v}=\frac{\epsilon_{v}}{\omega_{v}}$, where $\epsilon_{\mu}, \omega_{\mu}, \epsilon_{v}, \omega_{\nu} \in \mathbb{N}$. Then, we set $\xi=\kappa \cdot \operatorname{LCM}\left(\left\{\omega_{\mu}\right\},\left\{\omega_{\nu}\right\}\right)$, where $\kappa=1,2, \ldots, \xi-1$. By applying the $\alpha$-integral (the Riemann-Liouville fractional integral of order $\alpha$ ) to both sides of (30), we have

$$
\mathfrak{I}^{\alpha} D^{\alpha} \mathbf{x}(t)=\mathfrak{I}^{\alpha}\left\{\mathbf{A}(t) \mathbf{x}(t)+\mathbf{B}(t) \mathbf{u}(t)+\sum_{\mu=1}^{a} \mathbf{E}_{\mu}(t) \mathbf{x}\left(t-h_{\mu}\right)+\sum_{v=1}^{b} \mathbf{F}_{v}(t) \mathbf{u}\left(t-h_{v}\right)+\mathbf{G}(t) \mathbf{x}(1-t)+\mathbf{d}(t)\right\} .
$$

From (15), we have $\mathfrak{I}^{\alpha} D^{\alpha} \mathbf{x}(t)=\mathbf{x}(t)-\mathbf{x}(0)$. Using (4), $x_{i}(t)=\mathbf{\Psi}_{\tilde{\xi}}^{\top}(t) \mathbf{x}_{i c w}^{\top}$, where $i=1,2, \ldots, q$ and $\mathbf{x}_{i c w}^{\top}$ is a $\xi^{k-1} M \times 1$ vector of unknown parameters. From the property of Kronecker product, for $\mathbf{x}(t) \in \mathbb{R}^{q}$ we can write

$$
\mathbf{x}(t)=\left(\mathbf{\Psi}_{\xi}^{\top}(t) \otimes \mathbf{I}_{q}\right) \mathbf{X}_{c w} .
$$

Similarly,

$$
\mathbf{x}_{0}=\left(\mathbf{\Psi}_{\xi}^{\top}(t) \otimes \mathbf{I}_{q}\right) \mathbf{X}_{c w}^{0}
$$

so the left side of (32) becomes

$$
\mathfrak{I}^{\alpha} D^{\alpha} \mathbf{x}(t)=\left(\mathbf{\Psi}_{\mathcal{\zeta}}^{\top}(t) \otimes \mathbf{I}_{q}\right)\left\{\mathbf{X}_{c w}-\mathbf{X}_{c w}^{0}\right\}
$$

where $\mathbf{X}_{c w}$ is a $q \xi^{k-1} M \times 1$ column vector of unknown parameters, $\mathbf{X}_{c w}^{0}$ is a known $q \xi^{k-1} M \times 1$ column vector and they are defined as

$$
\begin{gathered}
\mathbf{X}_{c w}=\left[X_{10}^{\xi 1}, X_{10}^{\xi 2}, \ldots, X_{10}^{\xi q}, \ldots, X_{1 M-1}^{\xi 1}, X_{1 M-1}^{\xi 2}, \ldots, X_{1 M-1}^{\xi q}, \ldots, X_{\xi^{k-1} M-1}^{\xi 1}, X_{\xi^{\xi k-1} M-1}^{\xi 2}, \ldots, X_{\xi^{\xi k-1} M-1}^{\xi q}\right]^{\top}, \\
\mathbf{X}_{c w}^{0}=\frac{\sqrt{\pi}}{\sqrt{2 \xi^{k-1}}}\left[\mathbf{X}_{1}^{0}, \mathbf{X}_{2}^{0}, \ldots, \mathbf{X}_{\xi^{k-1}}^{0}\right]^{\top}, \mathbf{X}_{n}^{0}=[\mathbf{x}_{0}^{\top}, \overbrace{0,0, \ldots, 0}^{q(M-1)}] .
\end{gathered}
$$


We expand the control vector in terms of CWs as

$$
\mathbf{u}(t)=\left(\mathbf{\Psi}_{\xi}^{\top}(t) \otimes \mathbf{I}_{r}\right) \mathbf{U}_{c w}
$$

where $\mathbf{U}_{c w}$ is a $r \xi^{k-1} M \times 1$ column vector of unknown parameters and

$$
\mathbf{U}_{c w}=\left[U_{10}^{\xi 1}, U_{10}^{\xi 2}, \ldots, U_{10}^{\xi r}, \ldots, U_{1 M-1}^{\xi 1}, U_{1 M-1}^{\xi 2}, \ldots, U_{1 M-1}^{\xi r}, \ldots, U_{\xi^{k-1} M-1}^{\xi 1}, U_{\xi^{k-1} M-1}^{\xi 2}, \ldots, U_{\xi^{k-1} M-1}^{\xi r}\right]^{\top}
$$

The disturbance can be expanded as

$$
\mathbf{d}(t)=\left(\mathbf{\Psi}_{\xi}^{\top}(t) \otimes \mathbf{I}_{q}\right) \mathbf{d}_{c w}
$$

where

$$
\mathbf{d}_{c w}=\left[d_{10}^{\xi 1}, d_{10}^{\xi 2}, \ldots, d_{10}^{\xi q}, \ldots, d_{1 M-1}^{\xi 1}, d_{1 M-1}^{\xi 2}, \ldots, d_{1 M-1}^{\xi q}, \ldots, d_{\xi^{k-1} M-1}^{\xi 1}, d_{\xi^{k-1} M-1}^{\xi 2}, \ldots, d_{\xi^{k-1} M-1}^{\xi q}\right]^{\top} .
$$

Assume that $\mathbf{d}(t)=\left[d_{1}(t), d_{2}(t), \ldots, d_{q}(t)\right]^{\top}$. The coefficients $\left\{d_{n m}^{\tilde{\xi} v}\right\}$, where $v=1,2, \ldots, q$, are obtained from (5) as $d_{n m}^{\xi_{v}^{v}}=\frac{c_{m}}{\sqrt{2 \xi^{k-1}}} \int_{0}^{\pi} d_{v}\left(\frac{\cos \theta+2 n-1}{2 \xi^{k-1}}\right) \cos (m \theta) d \theta$.

We expand the time-varying matrices of the state equation in terms of CWs as

$$
\begin{gathered}
\mathbf{A}(t)=\mathbf{A}_{c w}\left(\mathbf{\Psi}_{\xi}(t) \otimes \mathbf{I}_{q}\right), \mathbf{E}_{\mu}(t)=\mathbf{E}_{\mu c w}\left(\mathbf{\Psi}_{\xi}(t) \otimes \mathbf{I}_{q}\right), \mathbf{G}(t)=\mathbf{G}_{c w}\left(\mathbf{\Psi}_{\xi}(t) \otimes \mathbf{I}_{q}\right), \\
\mathbf{B}(t)=\mathbf{B}_{c w}\left(\mathbf{\Psi}_{\xi}(t) \otimes \mathbf{I}_{r}\right), \mathbf{F}_{v}(t)=\mathbf{F}_{v c w}\left(\mathbf{\Psi}_{\xi}(t) \otimes \mathbf{I}_{r}\right) .
\end{gathered}
$$

$\mathbf{A}_{c w}, \mathbf{E}_{\mu c w}, \mathbf{G}_{c w}, \mathbf{B}_{c w}$, and $\mathbf{F}_{v c w}$ are constant matrices which can be determined by using (5). For detailed information, see [31]. In like manner, the initial functions can be expressed as

$$
\boldsymbol{\theta}\left(t-h_{\mu}\right)=\left(\mathbf{\Psi}_{\tilde{\xi}}^{\top}(t) \otimes \mathbf{I}_{q}\right) \boldsymbol{\theta}_{\mu c w}, \zeta\left(t-h_{\mu}\right)=\left(\mathbf{\Psi}_{\xi}^{\top}(t) \otimes \mathbf{I}_{r}\right) \zeta_{\mu c w}
$$

$\boldsymbol{\theta}_{\mu c w}, \zeta_{v c w}$ for $\mu=1,2, \ldots, a$ and $v=1,2, \ldots, b$ are $q \xi^{k-1} M \times 1$ and $r \xi^{k-1} M \times 1$ vectors defined by

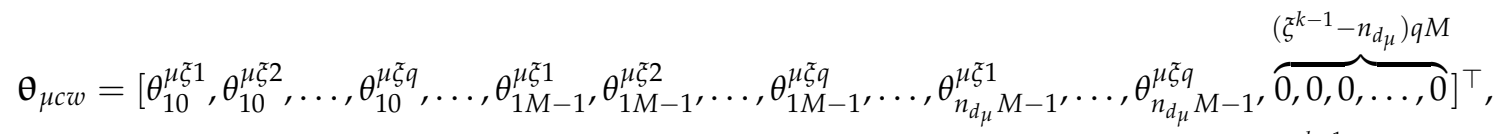

$$
\begin{aligned}
& \zeta_{v c w}=[\zeta_{10}^{v \xi 1}, \zeta_{10}^{v \zeta_{2}}, \ldots, \zeta_{10}^{v \xi r}, \ldots, \zeta_{1 M-1}^{v \zeta_{1}}, \zeta_{1 M-1}^{v \zeta_{2}}, \ldots, \zeta_{1 M-1}^{v \zeta^{2}}, \ldots, \zeta_{n_{d_{v}} M-1}^{v \xi_{1}}, \ldots, \zeta_{n_{d_{v}} M-1}^{v \xi_{r}}, \overbrace{0,0,0, \ldots, 0}^{\left(\xi^{k-1}-n_{d_{v}}\right) r M}]^{\top},
\end{aligned}
$$

where $n_{d_{\mu}}=h_{\mu} \xi^{k-1}$ and $n_{d_{v}}=h_{v} \xi^{k-1}$. From the conditions of initial functions, we have

$$
\begin{aligned}
& \mathbf{x}\left(t-h_{\mu}\right)=\boldsymbol{\theta}\left(t-h_{\mu}\right)+\left(\mathbf{\Psi}_{\xi}^{\top}\left(t-h_{\mu}\right) \otimes \mathbf{I}_{q}\right) \mathbf{X}_{c w}=\left(\mathbf{\Psi}_{\xi}^{\top}(t) \otimes \mathbf{I}_{q}\right)\left\{\boldsymbol{\theta}_{\mu c w}+\left(\mathbf{D}_{\mu c w}^{\top} \otimes \mathbf{I}_{q}\right) \mathbf{X}_{c w}\right\}, \\
& \mathbf{u}\left(t-h_{v}\right)=\zeta\left(t-h_{v}\right)+\left(\mathbf{\Psi}_{\xi}^{\top}\left(t-h_{v}\right) \otimes \mathbf{I}_{r}\right) \mathbf{U}_{c w}=\left(\mathbf{\Psi}_{\xi}^{\top}(t) \otimes \mathbf{I}_{r}\right)\left\{\boldsymbol{\zeta}_{v c w}+\left(\mathbf{D}_{v c w}^{\top} \otimes \mathbf{I}_{r}\right) \mathbf{U}_{c w}\right\} .
\end{aligned}
$$

For a matrix $\mathbf{O}_{i}$ of order $1 \times q M \xi^{k-1}$, we have $\mathbf{O}_{i}\left(\mathbf{\Psi}_{\xi}(t) \otimes \mathbf{I}_{q}\right)\left(\Psi_{\xi}^{\top}(t) \otimes \mathbf{I}_{q}\right)=\mathbf{\Psi}_{\xi}^{\top}(t) \tilde{\mathbf{O}}_{i}$, where $i=1,2, \ldots, q$. We form a matrix as $\mathbf{O}_{t}:=\left[\begin{array}{lll}\mathbf{O}_{1}^{\top} \mathbf{O}_{2}^{\top} \cdots & \mathbf{O}_{q}^{\top}\end{array}\right]^{\top}$, by considering the property of the product operational matrix, we see that $\mathbf{O}_{t}\left(\mathbf{\Psi}_{\xi}(t) \otimes \mathbf{I}_{q}\right)\left(\Psi_{\xi}^{\top}(t) \otimes \mathbf{I}_{q}\right)=\left(\mathbf{\Psi}_{\xi}^{\top}(t) \otimes \mathbf{I}_{q}\right) \tilde{\mathbf{O}}_{t}$. Additionally, for a $1 \times r M \xi^{k-1}$ matrix $\mathbf{O}_{i}$, we get $\mathbf{O}_{i}\left(\mathbf{\Psi}_{\xi}(t) \otimes \mathbf{I}_{r}\right)\left(\Psi_{\xi}^{\top}(t) \otimes \mathbf{I}_{r}\right)=\mathbf{\Psi}_{\xi}^{\top}(t) \tilde{\mathbf{O}}_{i}$. Similarly, by forming $\mathbf{O}_{t}$, we find $\mathbf{O}_{t}\left(\Psi_{\xi}(t) \otimes \mathbf{I}_{r}\right)\left(\Psi_{\xi}^{\top}(t) \otimes \mathbf{I}_{r}\right)=\left(\Psi_{\xi}^{\top}(t) \otimes \mathbf{I}_{q}\right) \tilde{\mathbf{O}}_{t}$. From these facts and the expressions given above, using (16) in the right-hand side of (32) and by (7)-(10) and (17) we can write 


$$
\begin{aligned}
& \mathfrak{I}^{\alpha}\left\{\mathbf{A}(t) \mathbf{x}(t)+\mathbf{B}(t) \mathbf{u}(t)+\sum_{\mu=1}^{a} \mathbf{E}_{\mu}(t) \mathbf{x}\left(t-h_{\mu}\right)+\sum_{v=1}^{b} \mathbf{F}_{v}(t) \mathbf{u}\left(t-h_{v}\right)+\mathbf{G}(t) \mathbf{x}(1-t)+\mathbf{d}(t)\right\} \\
& =\mathfrak{I}^{\alpha}\left\{\mathbf{A}_{c w}\left(\mathbf{\Psi}_{\tilde{\zeta}}(t) \otimes \mathbf{I}_{q}\right)\left(\mathbf{\Psi}_{\tilde{\zeta}}^{\top}(t) \otimes \mathbf{I}_{q}\right) \mathbf{X}_{c w}+\mathbf{B}_{c w}\left(\mathbf{\Psi}_{\xi}(t) \otimes \mathbf{I}_{r}\right)\left(\mathbf{\Psi}_{\mathcal{\zeta}}^{\top}(t) \otimes \mathbf{I}_{r}\right) \mathbf{U}_{c w}\right. \\
& +\sum_{\mu=1}^{a}\left(\mathbf{E}_{\mu c w}\left(\mathbf{\Psi}_{\xi}(t) \otimes \mathbf{I}_{q}\right)\left(\mathbf{\Psi}_{\mathcal{\zeta}}^{\top}(t) \otimes \mathbf{I}_{q}\right)\left\{\boldsymbol{\theta}_{\mu c w}+\left(\mathbf{D}_{\mu c w}^{\top} \otimes \mathbf{I}_{q}\right) \mathbf{X}_{c w}\right\}\right) \\
& +\sum_{v=1}^{b}\left(\mathbf{F}_{v c w}\left(\mathbf{\Psi}_{\xi}(t) \otimes \mathbf{I}_{r}\right)\left(\mathbf{\Psi}_{\xi}^{\top}(t) \otimes \mathbf{I}_{r}\right)\left\{\boldsymbol{\zeta}_{v c w}+\left(\mathbf{D}_{v c w}^{\top} \otimes \mathbf{I}_{r}\right) \mathbf{U}_{c w}\right\}\right) \\
& \left.+\mathbf{G}_{c w}\left(\mathbf{\Psi}_{\mathcal{\zeta}}(t) \otimes \mathbf{I}_{q}\right)\left(\mathbf{\Psi}_{\mathcal{\zeta}}^{\top}(t) \otimes \mathbf{I}_{q}\right)\left(\Upsilon_{c w}^{\top} \otimes \mathbf{I}_{q}\right) \mathbf{X}_{c w}+\left(\mathbf{\Psi}_{\tilde{\zeta}}^{\top}(t) \otimes \mathbf{I}_{q}\right) \mathbf{d}_{c w}\right\} \\
& =\mathfrak{I}^{\alpha}\left\{\boldsymbol{\Psi}_{\tilde{\zeta}}^{\top}(t) \otimes \mathbf{I}_{q}\right\}\left(\tilde{\mathbf{A}}_{c w} \mathbf{X}_{c w}+\tilde{\mathbf{B}}_{c w} \mathbf{U}_{c w}+\sum_{\mu=1}^{a}\left(\tilde{\mathbf{E}}_{\mu c w} \boldsymbol{\theta}_{\mu c w}+\tilde{\mathbf{E}}_{\mu c w}\left(\mathbf{D}_{\mu c w}^{\top} \otimes \mathbf{I}_{q}\right) \mathbf{X}_{c w}\right)+\sum_{v=1}^{b}\left(\tilde{\mathbf{F}}_{v c w} \zeta_{v c w}\right.\right. \\
& \left.\left.+\tilde{\mathbf{F}}_{v c w}\left(\mathbf{D}_{v c w}^{\top} \otimes \mathbf{I}_{r}\right) \mathbf{U}_{c w}\right)+\tilde{\mathbf{G}}_{c w}\left(\Upsilon_{c w}^{\top} \otimes \mathbf{I}_{q}\right) \mathbf{X}_{c w}+\mathbf{d}_{c w}\right) \\
& =\left(\mathbf{\Psi}_{\tilde{\zeta}}^{\top}(t) \otimes \mathbf{I}_{q}\right)\left(\mathbf{P}_{c w}^{\alpha}{ }^{\top} \otimes \mathbf{I}_{q}\right)\left(\tilde{\mathbf{A}}_{c w} \mathbf{X}_{c w}+\tilde{\mathbf{B}}_{c w} \mathbf{U}_{c w}+\sum_{\mu=1}^{a}\left(\tilde{\mathbf{E}}_{\mu c w} \boldsymbol{\theta}_{\mu c w}+\tilde{\mathbf{E}}_{\mu c w}\left(\mathbf{D}_{\mu c w}^{\top} \otimes \mathbf{I}_{q}\right) \mathbf{X}_{c w}\right)\right. \\
& \left.+\sum_{v=1}^{b}\left(\tilde{\mathbf{F}}_{v c w} \boldsymbol{\zeta}_{v c w}+\tilde{\mathbf{F}}_{v c w}\left(\mathbf{D}_{v c w}^{\top} \otimes \mathbf{I}_{r}\right) \mathbf{U}_{c w}\right)+\tilde{\mathbf{G}}_{c w}\left(\Upsilon_{c w}^{\top} \otimes \mathbf{I}_{q}\right) \mathbf{X}_{c w}+\mathbf{d}_{c w}\right),
\end{aligned}
$$

where we have denoted the product operational matrices of $\mathbf{A}_{c w}, \mathbf{B}_{c w}, \mathbf{E}_{\mu c w}, \mathbf{F}_{v c w}, \mathbf{G}_{c w}$ by, in turn, $\tilde{\mathbf{A}}_{c w}, \tilde{\mathbf{B}}_{c w}, \tilde{\mathbf{E}}_{\mu c w}, \tilde{\mathbf{F}}_{v c w}, \tilde{\mathbf{G}}_{c w}$. Now, the following compatibility constraint is imposed at $t_{\iota}$, where $\iota=$ $1,2, \ldots, \xi^{k-1}-1$, and $t_{l}=\iota / \xi^{k-1}$,

$$
\mathbf{x}\left(t_{l}^{-}\right)=\mathbf{x}\left(t_{l}^{+}\right)
$$

this constraint can be written

$$
\left(\mathbf{\Psi}_{c c} \otimes \mathbf{I}_{q}\right) \mathbf{X}_{c w}=\mathbf{0}_{\left(\tilde{s}^{k-1}-1\right) q \times 1}
$$



After substituting (33) and (34) in (32), and considering (35) we get

$$
\begin{gathered}
{\left[\begin{array}{c}
\left(\mathbf{P}_{c w}^{\alpha}{ }^{\top} \otimes \mathbf{I}_{q}\right) \tilde{\mathbf{A}}_{c w}+\sum_{\mu=1}^{a}\left(\mathbf{P}_{c w}^{\alpha} \top \otimes \mathbf{I}_{q}\right) \tilde{\mathbf{E}}_{\mu c w}\left(\mathbf{D}_{\mu c w}^{\top} \otimes \mathbf{I}_{q}\right)+\left(\mathbf{P}_{c w}^{\alpha}{ }^{\top} \otimes \mathbf{I}_{q}\right) \tilde{\mathbf{G}}_{c w}\left(\Upsilon_{c w}^{\top} \otimes \mathbf{I}_{q}\right)-\mathbf{I}_{q s^{k-1} M} \\
\mathbf{\Psi}_{c c} \otimes \mathbf{I}_{q} \\
\left(\mathbf{P}_{c w}^{\alpha}{ }^{\top} \otimes \mathbf{I}_{q}\right) \tilde{\mathbf{B}}_{c w}+\sum_{v=1}^{b}\left(\mathbf{P}_{c w}^{\alpha}{ }^{\top} \otimes \mathbf{I}_{q}\right) \tilde{\mathbf{F}}_{v c w}\left(\mathbf{D}_{v c w}^{\top} \otimes \mathbf{I}_{r}\right) \\
\mathbf{0}
\end{array}\right]\left[\begin{array}{c}
\mathbf{X}_{c w} \\
\mathbf{U}_{c w}
\end{array}\right]} \\
=\left[\begin{array}{c}
-\mathbf{X}_{c w}^{0}-\left(\mathbf{P}_{c w}^{\alpha}{ }^{\top} \otimes \mathbf{I}_{q}\right) \mathbf{d}_{c w}-\sum_{\mu=1}^{a}\left(\mathbf{P}_{c w}^{\alpha}{ }^{\top} \otimes \mathbf{I}_{q}\right) \tilde{\mathbf{E}}_{\mu c w} \boldsymbol{\theta}_{\mu c w}-\sum_{v=1}^{b}\left(\mathbf{P}_{c w}^{\alpha}{ }^{\top} \otimes \mathbf{I}_{q}\right) \tilde{\mathbf{F}}_{v c w} \zeta_{v c w} \\
\mathbf{0}
\end{array}\right] .
\end{gathered}
$$

Setting

$$
\mathbf{Q}(t)=\mathbf{Q}_{c w}\left(\mathbf{\Psi}_{\xi}(t) \otimes \mathbf{I}_{q}\right), \mathbf{R}(t)=\mathbf{R}_{c w}\left(\mathbf{\Psi}_{\xi}(t) \otimes \mathbf{I}_{r}\right)
$$

in (31), and using (7) and (11) yields

$$
J=\frac{1}{2}\left[\begin{array}{c}
\mathbf{X}_{c w} \\
\mathbf{U}_{c w}
\end{array}\right]^{\top}\left[\begin{array}{cc}
\mathbf{\Psi}_{\xi}(1) \mathbf{\Psi}_{\tilde{\zeta}}^{\top}(1) \otimes \mathbf{T}+\left(\boldsymbol{\Gamma}_{c w} \otimes \mathbf{I}_{q}\right) \tilde{\mathbf{Q}}_{c w} & \mathbf{0} \\
\mathbf{0} & \left(\boldsymbol{\Gamma}_{c w} \otimes \mathbf{I}_{r}\right) \tilde{\mathbf{R}}_{c w}
\end{array}\right]\left[\begin{array}{c}
\mathbf{X}_{c w} \\
\mathbf{U}_{c w}
\end{array}\right]
$$

where we have denoted the product operational matrices of $\mathbf{Q}_{c w}$ and $\mathbf{R}_{c w}$ by, in turn, $\tilde{\mathbf{Q}}_{c w}$ and $\tilde{\mathbf{R}}_{c w}$. 
Finally, from (36), (37), and $\mathbf{\Psi}_{\xi \mathcal{C} C}:=\left[\begin{array}{c}\mathbf{\Psi}_{c c} \\ \mathbf{\Psi}_{\tilde{\zeta}}^{\top}(0)\end{array}\right]$, the fractional time-delay optimal control problem is

$$
\min \frac{1}{2}\left[\begin{array}{c}
\mathbf{X}_{c w} \\
\hdashline \mathbf{U}_{c w}^{-}
\end{array}\right]^{\top}\left[\begin{array}{c:c}
\Xi_{1} & \Xi_{2} \\
\hdashline \Xi_{3} & \Xi_{4}^{-}
\end{array}\right]\left[\begin{array}{c}
\mathbf{X}_{c w} \\
\hdashline \mathbf{U}_{c w}^{-}
\end{array}\right],
$$

$$
\text { subject to } \quad\left[\begin{array}{c:c}
\boldsymbol{\Lambda}_{1} & \boldsymbol{\Lambda}_{2} \\
\hdashline \boldsymbol{\Lambda}_{3} & \boldsymbol{\Lambda}_{4}
\end{array}\right]\left[\begin{array}{c}
\mathbf{X}_{c w} \\
\hdashline \mathbf{U}_{c w}^{-}
\end{array}\right]=\left[\begin{array}{c}
\mathbf{b}_{1} \\
\hdashline \mathbf{b}_{2}
\end{array}\right],
$$

where



$$
\begin{aligned}
& \mathbf{\Lambda}_{1}=\left(\mathbf{P}_{c w}^{\alpha}{ }^{\top} \otimes \mathbf{I}_{q}\right) \tilde{\mathbf{A}}_{c w}+\sum_{\mu=1}^{a}\left(\mathbf{P}_{c w}^{\alpha} \tilde{\mathbf{B}}^{\top} \otimes \mathbf{I}_{q}\right) \tilde{\mathbf{E}}_{\mu c w}\left(\mathbf{D}_{\mu c w}^{\top} \otimes \mathbf{I}_{q}\right)+\left(\mathbf{P}_{c w}^{\alpha}{ }^{\top} \otimes \mathbf{I}_{q}\right) \tilde{\mathbf{G}}_{c w}\left(\Upsilon_{c w}^{\top} \otimes \mathbf{I}_{q}\right)-\mathbf{I}_{q s^{z k-1} M^{\prime}} \\
& \boldsymbol{\Lambda}_{2}=\left(\mathbf{P}_{c w}^{\alpha}{ }^{\top} \otimes \mathbf{I}_{q}\right) \tilde{\mathbf{B}}_{c w}+\sum_{v=1}^{b}\left(\mathbf{P}_{c w}^{\alpha}{ }^{\top} \otimes \mathbf{I}_{q}\right) \tilde{\mathbf{F}}_{v c w}\left(\mathbf{D}_{v c w}^{\top} \otimes \mathbf{I}_{r}\right), \\
& \Lambda_{3}=\mathbf{\Psi}_{\xi \mathcal{C}} \otimes \mathbf{I}_{q} \\
& \mathbf{\Lambda}_{4}=\mathbf{0}_{q \xi^{k-1} \times r \xi^{k-1} M^{\prime}} \\
& \left.\begin{array}{l}
\mathbf{b}_{1}=-\mathbf{X}_{c w}^{0}-\left(\mathbf{P}_{c w}^{\alpha}{ }^{\top} \otimes \mathbf{I}_{q}\right) \mathbf{d}_{c w}-\sum_{\mu=1}^{a}\left(\mathbf{P}_{c w}^{\alpha}{ }^{\top} \otimes \mathbf{I}_{q}\right) \tilde{\mathbf{E}}_{\mu c w} \boldsymbol{\theta}_{\mu c w}-\sum_{v=1}^{b}\left(\mathbf{P}_{c w}^{\alpha}{ }^{\top} \otimes \mathbf{I}_{q}\right) \tilde{\mathbf{F}}_{v c w} \zeta_{v c w}, \\
\mathbf{b}_{2}=\left[\begin{array}{c}
\mathbf{0}_{q\left(\xi^{k-1}-1\right) \times 1} \\
\mathbf{x}_{0}
\end{array}\right] .
\end{array}\right\}
\end{aligned}
$$

So, fractional linear-quadratic delay optimal control problems are converted to QP problems which as we shall see later (without doing any significant work) by constructing (40)-(42) and putting into (38) and (39) we are able to solve such problems.

Next, we extend the CW method to the system in which the fractional order is greater than 1 .

B. $1<\alpha \leq 2$

This causes a change in (33) or the left side of (32); using (15), in which $n=2$, we find that

$$
\begin{aligned}
\mathfrak{I}^{\alpha} D^{\alpha} \mathbf{x}(t) & =\mathbf{x}(t)-\mathbf{x}(0)-\left(t \otimes \mathbf{I}_{q}\right) \dot{\mathbf{x}}(0) \\
& =\left(\mathbf{\Psi}_{\tilde{\mathcal{S}}}^{\top}(t) \otimes \mathbf{I}_{q}\right) \mathbf{X}_{c w}-\left(\mathbf{\Psi}_{\tilde{\zeta}}^{\top}(t) \otimes \mathbf{I}_{q}\right) \mathbf{X}_{c w}^{0}-\left(\mathbf{\Psi}_{\tilde{\zeta}}^{\top}(t) \otimes \mathbf{I}_{q}\right)\left(\boldsymbol{\tau}_{c w} \otimes \mathbf{I}_{q}\right) \dot{\mathbf{x}}(0) \\
& =\left(\mathbf{\Psi}_{\tilde{\zeta}}^{\top}(t) \otimes \mathbf{I}_{q}\right)\left\{\mathbf{X}_{c w}-\mathbf{X}_{c w}^{0}-\left(\boldsymbol{\tau}_{c w} \otimes \mathbf{I}_{q}\right) \dot{\mathbf{x}}(0)\right\},
\end{aligned}
$$

where we have set

$$
t=\mathbf{\Psi}_{\tilde{\zeta}}^{\top}(t) \boldsymbol{\tau}_{c w}
$$

and $\dot{\mathbf{x}}(0)=\mathbf{x}^{(1)}(0)$. (43) has the effect of changing the matrix $\mathbf{b}_{1}$. Therefore, $\mathbf{b}_{1}$ in (42) is changed to

$$
\mathbf{b}_{1}=-\mathbf{X}_{c w}^{0}-\left(\boldsymbol{\tau}_{c w} \otimes \mathbf{I}_{q}\right) \dot{\mathbf{x}}(0)-\left(\mathbf{P}_{c w}^{\alpha}{ }^{\top} \otimes \mathbf{I}_{q}\right) \mathbf{d}_{c w}-\sum_{\mu=1}^{a}\left(\mathbf{P}_{c w}^{\alpha}{ }^{\top} \otimes \mathbf{I}_{q}\right) \tilde{\mathbf{E}}_{\mu c w} \boldsymbol{\theta}_{\mu c w}-\sum_{v=1}^{b}\left(\mathbf{P}_{c w}^{\alpha}{ }^{\top} \otimes \mathbf{I}_{q}\right) \tilde{\mathbf{F}}_{v c w} \zeta_{v c w} .
$$

The other matrices are the same as before and just by changing $\mathbf{b}_{1}$ given in (45) we solve the problem.

\subsection{Analysis of Linear Fractional Time-Delay Systems}

Now, we present a method for the analysis of linear systems modeled by multifractional delay differential equations. Indeed, the following discussion permits an extension of the method to multifractional optimal control problems. Consider a general linear system modeled by

$$
D^{\alpha_{1}} \mathbf{x}(t)+\mathbf{\Omega}_{1} D^{\alpha_{2}} \mathbf{x}(t)+\mathbf{\Omega}_{2} D^{\alpha_{3}} \mathbf{x}(t-h)=\mathbf{A}(t) \mathbf{x}(t)+\mathbf{B}(t) \mathbf{u}(t)+\mathbf{C}(t) \mathbf{x}(t-h(t))+\sum_{\mu=1}^{a} \mathbf{E}_{\mu}(t) \mathbf{x}\left(t-h_{\mu}\right),
$$


where $1<\alpha_{1} \leq 2,0<\alpha_{2} \leq 1,0<\alpha_{3} \leq 1, t \in[0,1], \mathbf{x}(t)$ is $q$ th state vector, $\mathbf{u}(t)$ is $r$ th input vector, $\boldsymbol{\Omega}_{1}, \boldsymbol{\Omega}_{2}, \mathbf{A}(t), \mathbf{B}(t), \mathbf{C}(t)$, and $\mathbf{E}_{\mu}(t)$ are matrices, $h$ and $\left\{h_{\mu}\right\}, \mu=1,2, \ldots, a$ are delays, and $h(t)$ is a piecewise constant delay. The initial condition and function are

$$
\mathbf{x}^{(i)}(0)=\mathbf{x}_{i}, \mathbf{x}(t)=\theta(t), \quad t<0 .
$$

The problem is to find the response of the given fractional time-delay system.

Assume that $\Omega_{1}$ and $\Omega_{2}$ are time-invariant matrices. Similar to Section 4.1, we expand $\mathbf{x}(t), \mathbf{x}_{0}$, $\mathbf{A}(t), \mathbf{B}(t), \mathbf{E}_{\mu}(t), \boldsymbol{\theta}\left(t-h_{\mu}\right)$, and $\mathbf{u}(t)$ in terms of CWs ( $\mathbf{U}_{c w}$ in known). In addition, we express $\mathbf{C}(t)$ and $\boldsymbol{\theta}(t-h(t))$ as $\mathbf{C}(t)=\mathbf{C}_{c w}\left(\Psi_{\xi}(t) \otimes \mathbf{I}_{q}\right)$ and $\boldsymbol{\theta}(t-h(t))=\left(\boldsymbol{\Psi}_{\tilde{\zeta}}^{\top}(t) \otimes \mathbf{I}_{q}\right) \boldsymbol{\theta}_{c w}^{t}$, where $\boldsymbol{\theta}_{c w}^{t}$ was defined in [28]. Moreover, we can write $\boldsymbol{\theta}(t-h)=\left(\mathbf{\Psi}_{\tilde{\zeta}}^{\top}(t) \otimes \mathbf{I}_{q}\right) \boldsymbol{\theta}_{c w}$ and $\boldsymbol{\theta}(-h)=\left(\mathbf{\Psi}_{\tilde{\xi}}^{\top}(t) \otimes \mathbf{I}_{q}\right) \boldsymbol{\theta}_{c w}^{0}$. For $h$ and $h(t)$, we have two delay operational matrices as $\mathbf{D}_{c w}$ and $\mathbf{D}_{c w}^{t}$. Using the Riemann-Liouville fractional integral (order $\alpha_{1}$ ) on the left side of (46), substituting the given expressions and using (14) now yield

$$
\begin{aligned}
& \mathfrak{I}^{\alpha_{1}}\left\{D^{\alpha_{1}} \mathbf{x}(t)+\mathbf{\Omega}_{1} D^{\alpha_{2}} \mathbf{x}(t)+\mathbf{\Omega}_{2} D^{\alpha_{3}} \mathbf{x}(t-h)\right\} \\
& =\mathbf{x}(t)-\mathbf{x}(0)-\left(t \otimes \mathbf{I}_{q}\right) \dot{\mathbf{x}}(0)+\mathfrak{I}^{\alpha_{1}-\alpha_{2}}\left[\mathfrak{I}^{\alpha_{2}}\left\{\boldsymbol{\Omega}_{1} D^{\alpha_{2}} \mathbf{x}(t)\right\}\right]+\mathfrak{I}^{\alpha_{1}-\alpha_{3}}\left[\mathfrak{I}^{\alpha_{3}}\left\{\boldsymbol{\Omega}_{2} D^{\alpha_{3}} \mathbf{x}(t-h)\right\}\right] \\
& =\left(\mathbf{\Psi}_{\xi}^{\top}(t) \otimes \mathbf{I}_{q}\right)\left[\mathbf{X}_{c w}-\mathbf{X}_{c w}^{0}-\left(\boldsymbol{\tau}_{c w} \otimes \mathbf{I}_{q}\right) \dot{\mathbf{x}}(0)\right]+\mathfrak{I}^{\alpha_{1}-\alpha_{2}}\left(\mathbf{\Psi}_{\tilde{\zeta}}^{\top}(t) \otimes \mathbf{\Omega}_{1}\right)\left[\mathbf{X}_{c w}-\mathbf{X}_{c w}^{0}\right] \\
& +\mathfrak{I}^{\alpha_{1}-\alpha_{3}}\left(\mathbf{\Psi}_{\tilde{\zeta}}^{\top}(t) \otimes \mathbf{\Omega}_{2}\right)\left[\boldsymbol{\theta}_{c w}-\theta_{c w}^{0}+\left(\mathbf{D}_{c w}^{\top} \otimes \mathbf{I}_{q}\right) \mathbf{X}_{c w}\right] \\
& =\left(\mathbf{\Psi}_{\tilde{\zeta}}^{\top}(t) \otimes \mathbf{I}_{q}\right)\left\{\mathbf{X}_{c w}-\mathbf{X}_{c w}^{0}-\left(\boldsymbol{\tau}_{c w} \otimes \mathbf{I}_{q}\right) \dot{\mathbf{x}}(0)+\left(\mathbf{P}_{c w}^{\alpha_{1}-\alpha_{2} \top} \otimes \mathbf{\Omega}_{1}\right) \mathbf{X}_{c w}-\left(\mathbf{P}_{c w}^{\alpha_{1}-\alpha_{2} \top} \otimes \mathbf{\Omega}_{1}\right) \mathbf{X}_{c w}^{0}\right. \\
& \left.+\left(\mathbf{P}_{c w}^{\alpha_{1}-\alpha_{3} \top} \otimes \mathbf{\Omega}_{2}\right)\left(\mathbf{D}_{c w}^{\top} \otimes \mathbf{I}_{q}\right) \mathbf{X}_{c w}+\left(\mathbf{P}_{c w}^{\alpha_{1}-\alpha_{3} \top} \otimes \mathbf{\Omega}_{2}\right) \boldsymbol{\theta}_{c w}-\left(\mathbf{P}_{c w}^{\alpha_{1}-\alpha_{3} \top} \otimes \mathbf{\Omega}_{2}\right) \theta_{c w}^{0}\right\},
\end{aligned}
$$

where $\boldsymbol{\tau}_{c w}$ is defined in (44). Moreover, by the same procedure in the right side of (46), we find

$$
\begin{aligned}
\mathfrak{I}^{\alpha_{1}}\{\mathbf{A}(t) \mathbf{x}(t)+\mathbf{B}(t) \mathbf{u}(t)+\mathbf{C}(t) \mathbf{x}(t-h(t)) & \left.+\sum_{\mu=1}^{a} \mathbf{E}_{\mu}(t) \mathbf{x}\left(t-h_{\mu}\right)\right\} \\
=\left(\mathbf{\Psi}_{\tilde{\zeta}}^{\top}(t) \otimes \mathbf{I}_{q}\right)\left(\mathbf{P}_{c w}^{\alpha_{1}}{ }^{\top} \otimes \mathbf{I}_{q}\right)\left(\tilde{\mathbf{A}}_{c w} \mathbf{X}_{c w}+\right. & \tilde{\mathbf{B}}_{c w} \mathbf{U}_{c w}+\tilde{\mathbf{C}}_{c w} \boldsymbol{\theta}_{c w}^{t}+\tilde{\mathbf{C}}_{c w}\left(\mathbf{D}_{c w}^{t}{ }^{\top} \otimes \mathbf{I}_{q}\right) \mathbf{X}_{c w} \\
& \left.+\sum_{\mu=1}^{a}\left(\tilde{\mathbf{E}}_{\mu c w} \boldsymbol{\theta}_{\mu c w}+\tilde{\mathbf{E}}_{\mu c w}\left(\mathbf{D}_{\mu c w}^{\top} \otimes \mathbf{I}_{q}\right) \mathbf{X}_{c w}\right)\right) .
\end{aligned}
$$

Then, by (47) and (48),

$$
\begin{gathered}
\mathbf{X}_{c w}=\left[\mathbf{I}_{q \xi^{k-1} M}+\left(\mathbf{P}_{c w}^{\alpha_{1}-\alpha_{2} \top} \otimes \mathbf{\Omega}_{1}\right)+\left(\mathbf{P}_{c w}^{\alpha_{1}-\alpha_{3} \top} \otimes \mathbf{\Omega}_{2}\right)\left(\mathbf{D}_{c w}^{\top} \otimes \mathbf{I}_{q}\right)-\left(\mathbf{P}_{c w}^{\alpha_{1} \top} \otimes \mathbf{I}_{q}\right) \tilde{\mathbf{A}}_{c w}\right. \\
\left.-\left(\mathbf{P}_{c w}^{\alpha_{1} \top} \otimes \mathbf{I}_{q}\right) \tilde{\mathbf{C}}_{c w}\left(\mathbf{D}_{c w}^{t}{ }^{\top} \otimes \mathbf{I}_{q}\right)-\sum_{\mu=1}^{a}\left(\mathbf{P}_{c w}^{\alpha_{1} \top} \otimes \mathbf{I}_{q}\right) \tilde{\mathbf{E}}_{\mu c w}\left(\mathbf{D}_{\mu c w}^{\top} \otimes \mathbf{I}_{q}\right)\right]^{-1} \\
{\left[\mathbf{X}_{c w}^{0}+\left(\boldsymbol{\tau}_{c w} \otimes \mathbf{I}_{q}\right) \dot{\mathbf{x}}(0)+\left(\mathbf{P}_{c w}^{\alpha_{1}-\alpha_{2} \top} \otimes \mathbf{\Omega}_{1}\right) \mathbf{X}_{c w}^{0}-\left(\mathbf{P}_{c w}^{\alpha_{1}-\alpha_{3} \top} \otimes \mathbf{\Omega}_{2}\right) \boldsymbol{\theta}_{c w}+\left(\mathbf{P}_{c w}^{\alpha_{1}-\alpha_{3} \top} \otimes \mathbf{\Omega}_{2}\right) \boldsymbol{\theta}_{c w}^{0}\right.} \\
+\left(\left(\mathbf{P}_{c w}^{\alpha_{1} \top} \otimes \mathbf{I}_{q}\right) \tilde{\mathbf{B}}_{c w} \mathbf{U}_{c w}+\left(\mathbf{P}_{c w}^{\alpha_{1} \top} \otimes \mathbf{I}_{q}\right) \tilde{\mathbf{C}}_{c w} \boldsymbol{\theta}_{c w}^{t}+\sum_{\mu=1}^{a}\left(\mathbf{P}_{c w}^{\alpha_{1} \top} \otimes \mathbf{I}_{q}\right) \tilde{\mathbf{E}}_{\mu c w} \boldsymbol{\theta}_{\mu c w}\right],
\end{gathered}
$$

where $\mathbf{X}_{c w}$ is the solution of the fractional-order delay differential equation in the form of CWs.

We can summarize these methods in two given algorithms like those in [28].

\section{Illustrative Examples}

In this section, we are going to use the new fractional integration operational matrix of Chebyshev wavelets to some fractional systems. We shall give some tests for the accuracy of the new formulation. It has already been mentioned in [33] that the proposed method (modeling a linear-quadratic optimal control problem as a QP) has good future and high degree of flexibility. We have considered different kinds of conditions and constraints in the previous works, and we can solve the fractional 
linear-quadratic optimal control problem in such situations. But to show the flexibility of the method, we will consider different kinds of constraints.

\subsection{Example 1}

We are interested in finding the optimal control and state which, when applied to the fractional time-delay system, are expressed by

$$
\begin{gathered}
D^{\alpha} x(t)=-x(t)+x\left(t-\frac{1}{3}\right)+u(t)-0.5 u\left(t-\frac{2}{3}\right), \quad 0 \leq t \leq 1 \\
\left\{\begin{array}{l}
\theta(t)=1, \quad-\frac{1}{3} \leq t<0 \\
\zeta(t)=0, \quad-\frac{2}{3} \leq t \leq 0
\end{array}\right.
\end{gathered}
$$

minimize the cost function

$$
J=\frac{1}{2} \int_{0}^{1}\left\{x^{2}(t)+\frac{1}{2} u^{2}(t)\right\} d t
$$

where $x(0)=1$ and $x^{(1)}(0)=-0.1$.

We select $\xi=3$, setting $k=2$ and $M=3$ gives

$$
\begin{aligned}
& \mathbf{P}_{c w}=\frac{1}{6^{\alpha}}\left[\begin{array}{ccc}
\mathbf{Y}^{\alpha} & \mathbf{J}_{1}^{\alpha} & \mathbf{J}_{2}^{\alpha} \\
0 & \mathbf{Y}^{\alpha} & \mathbf{J}_{1}^{\alpha} \\
0 & 0 & \mathbf{Y}^{\alpha}
\end{array}\right], \boldsymbol{\Gamma}_{c w}=\frac{2}{\pi} \cdot \operatorname{blkdiag}(\boldsymbol{\Gamma}, \boldsymbol{\Gamma}, \boldsymbol{\Gamma}), \mathbf{D}_{\mu c w}=\left[\begin{array}{c}
\mathbf{D}_{1} \\
\mathbf{0}_{3 \times 9}
\end{array}\right], \mathbf{D}_{v c w}=\left[\begin{array}{c}
\mathbf{D}_{2} \\
\mathbf{0}_{6 \times 9}
\end{array}\right], \\
& \mathbf{D}_{1}=\left[\mathbf{0}_{6 \times 3} \mathbf{I}_{6}\right], \mathbf{D}_{2}=\left[\mathbf{0}_{3 \times 6} \mathbf{I}_{3}\right], \mathbf{X}_{c w}^{0}=\sqrt{\frac{\pi}{6}}[1,0,0,1,0,0,1,0,0]^{\top}, \boldsymbol{\theta}_{\mu c w}=\left[\sqrt{\frac{\pi}{6}}, 0,0,0,0,0,0,0,0\right]^{\top} .
\end{aligned}
$$

For $\alpha=1,0.999$, we found, in turn, $J^{*}=0.37313$ and $J^{*}=0.373042$ ( $*$ indicates optimal condition). Choosing $M=7$, of the optimal cost $J^{*}$, are shown in Table 1 . This fractional optimal control problem has been studied in some texts as [18-20]. By using the QP solver in MATLAB, we can easily solve the problem for various values of $\alpha$. By doing this, the comparison is made in Table 1 and one can see the accuracy of the method which provides a similar result as that we have found by Chebyshev wavelets [28] in the case $\alpha=1$. Additionally, we solve this problem for $\alpha>1$; as a test for the new

\begin{tabular}{|c|c|c|c|c|c|c|}
\hline$\alpha$ & This Work; $k=2, M=7$ & {$[28] ; k=2, M=7$} & [34]; $k=2, M=7$ & [18] & [19] & [20] \\
\hline 1 & 0.37311293528 & 0.373112935096 & 0.373112935279 & 0.01451 & 0.04553 & 0.37311264 \\
\hline 0.999 & 0.37302124305 & & & 0.01450 & & \\
\hline 0.99 & 0.37219761493 & & & 0.01436 & & 0.3721964 \\
\hline 0.95 & 0.36856850562 & & & & & 0.3685506 \\
\hline 0.9 & 0.36409192174 & & & 0.01336 & & 0.3640344 \\
\hline 0.89 & 0.36320335165 & & & & & \\
\hline 0.8 & 0.35528976948 & & & 0.01314 & & 0.3551193 \\
\hline 0.7 & 0.34662823700 & & & & & 0.3463065 \\
\hline 0.5 & 0.32938391796 & & & & & \\
\hline
\end{tabular}
fractional integration operational matrix of CWs, we apply Lemma 1 in (41) and (42): Strategy 1, we use $\mathbf{P}_{c w}^{\top} \mathbf{P}_{c w}^{\alpha-1}{ }^{\top}$; and Strategy 2, we use directly $\mathbf{P}_{c w}^{\alpha}{ }^{\top}$. The results are reported in Table 2. Comparing the results of the test shows the accuracy of the new matrix.

Table 1. Comparison of $J^{*}$ for $0.5 \leq \alpha \leq 1$, Example 1 .

Table 2. $J^{*}$ for $\alpha>1$, Example 1 .

\begin{tabular}{lll}
\hline $\boldsymbol{\alpha}$ & $\boldsymbol{J}^{*}$, Strategy $\mathbf{1}$ & $\boldsymbol{J}^{*}$, Strategy 2 \\
\hline 1.001 & 0.34564307806 & 0.34564144998 \\
1.01 & 0.34638051376 & 0.34636577766 \\
1.05 & 0.34965701531 & 0.34961784656 \\
1.1 & 0.35375919815 & 0.35376402419 \\
1.11 & 0.35458119323 & 0.35460259170 \\
\hline
\end{tabular}


The optimal cost in (50) indicates the total system energy [35] by equating the state weighting matrix to zero for $\alpha=1,0.9$, we have $\int_{0}^{1} \frac{1}{4} u^{*^{2}}(t) d t=0.08987169038,0.09433894184, \int_{0}^{h_{v}} \frac{1}{4} u^{*^{2}}(t) d t=$ $0.08533015864,0.08831955446$, respectively. Now, we consider constraints on control energy as

Case 1: $\quad \int_{0}^{1} \frac{1}{4} u^{2}(t) d t \leq 0.05$.

Case 2: $\int_{0}^{h_{v}} \frac{1}{4} u^{2}(t) d t \leq 0.05$, where $h_{v}=\frac{2}{3}$.

Proceeding as we did for approximating the cost function in (31), we model these problems by quadratically-constrained quadratic programs, then we can use the Toolbox introduced in [36]. The optimal controls and states obtained by this Toolbox for some $\alpha$ are shown in Figure 1. The values of the optimal cost $J^{*}$ are given in Table 3. In addition, to get a feel for the accuracy of the method, the values of $g_{1}:=\int_{0}^{1} \frac{1}{4} u^{*^{2}}(t) d t$ and $g_{2}:=\int_{0}^{h_{v}} \frac{1}{4} u^{*^{2}}(t) d t$ are given in this table. Since the optimal control of the unconstrained system does not satisfy both the constraints, we take $e_{1}=0.05-g_{1}$ and $e_{2}=0.05-g_{2}$ as criteria for the accuracy which must be small. We find that $e_{1}, e_{2} \leq 4.82 E-08$.
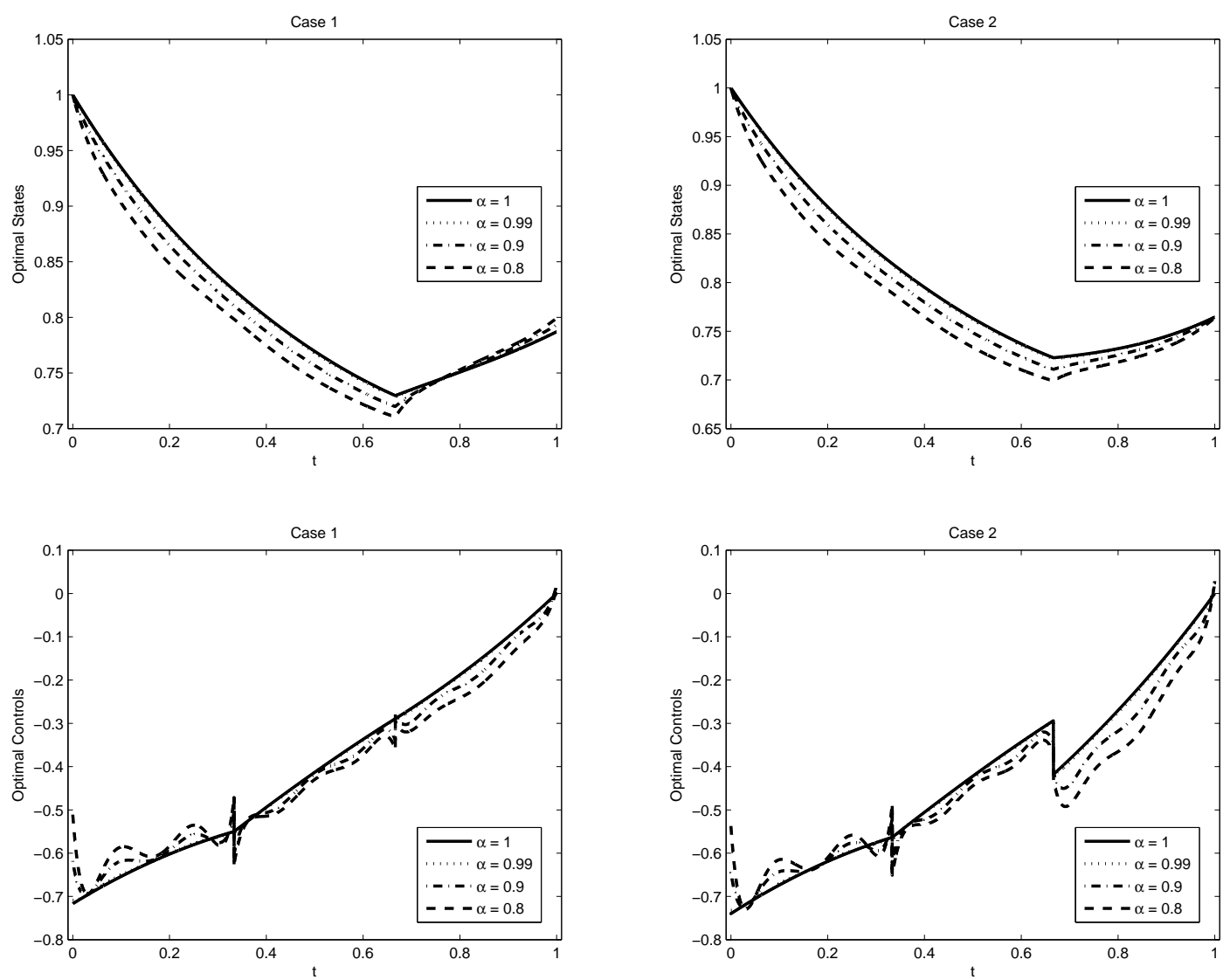

Figure 1. Optimal states and controls for Cases 1 and 2, Example 1.

Table 3. Numerical results for Cases 1 and 2, Example 1.

\begin{tabular}{|c|c|c|c|c|c|c|}
\hline \multicolumn{3}{|c|}{ Case 1} & \multicolumn{4}{|c|}{ Case 2} \\
\hline$\alpha$ & $J^{*}$ & $g_{1}$ & $\alpha$ & $J^{*}$ & $g_{2}$ & $g_{1}$ \\
\hline 1 & 0.38129264275 & 0.0499999578 & 1 & 0.37958583678 & 0.0499999519 & 0.0553443427 \\
\hline 0.999 & 0.38121931108 & 0.0499999578 & 0.999 & 0.37950598319 & 0.0499999519 & 0.0553602137 \\
\hline 0.99 & 0.38056110670 & 0.0499999583 & 0.99 & 0.37878828547 & 0.0499999523 & 0.0555045828 \\
\hline 0.9 & 0.37412772028 & 0.0499999624 & 0.9 & 0.37167971572 & 0.0499999560 & 0.0570967526 \\
\hline 0.8 & 0.36721704588 & 0.0499999659 & 0.8 & 0.36383702347 & 0.0499999589 & 0.0591798411 \\
\hline
\end{tabular}




\subsection{Example 2}

The problem is minimizing

$$
J=\int_{0}^{2}\left\{x^{2}(t)+u^{2}(t)\right\} d t
$$

subject to

$$
\begin{gathered}
D^{\alpha} x(t)=t x(t)+x(t-1)+u(t)+\mathfrak{a}(0.2-0.15 \cos t), 0 \leq t \leq 2 \\
\theta(t)=1,-1 \leq t<0,
\end{gathered}
$$

where $x(0)=1$ and $\dot{x}(0)=0.05$. Additionally, $\mathfrak{a}=0,1$ and we may have the disturbance in the system.

Setting $t / 2 \longrightarrow t$, the state equation is rescaled as

$$
D^{\alpha} x(t)=2^{\alpha}\left\{2 t x(t)+x\left(t-\frac{1}{2}\right)+u(t)+\mathfrak{a}(0.2-0.15 \cos 2 t)\right\}, 0 \leq t \leq 1 .
$$

We apply the method on this problem for different values of $\alpha$. We begin with the case in which $\alpha \leq 1$ and $\mathfrak{a}=0$ (with no disturbance) to compare the results of the method. Additionally, for $\alpha \leq 1$ and $\alpha>1$, we solve the problem with $\mathfrak{a}=1$, where the disturbance affects the plant dynamics. This situation may arise when control systems are subjected to external disturbances. We also solve the problem by the methods presented in [28] and [34] (integer values of $\alpha$ ). Our results are given in Table 4 . For some values of $\alpha \geq 1$, the results of applying the method to the system are shown in Figure 2. We can see the results of applying the new method presented for fractional optimization

\begin{tabular}{|c|c|c|c|c|c|c|c|}
\hline $\mathfrak{a}$ & $\alpha$ & This Work, $k=2, M=7$ & [28] $k=2, M=7$ & [34] $k=2, M=7$ & [20] & [37] & {$[38]$} \\
\hline 0 & 1 & 4.79679791916 & 4.79679791913 & 4.79679870920 & 4.79679868 & 4.7968 & 4.796817 \\
\hline 0 & 0.999 & 4.79697117915 & & & & & \\
\hline 0 & 0.99 & 4.79853220259 & & & 4.7766443 & & \\
\hline 0 & 0.95 & 4.80544625813 & & & 4.6907801 & & \\
\hline 0 & 0.9 & 4.81377758646 & & & 4.5728139 & & \\
\hline 0 & 0.8 & 4.82825064845 & & & 4.3096610 & & \\
\hline 0 & 0.7 & 4.83888850210 & & & 4.0256671 & & \\
\hline 0 & 0.5 & 4.84814116845 & & & & & \\
\hline 1 & 0.9 & 5.27371052428 & & & & & \\
\hline 1 & 0.99 & 5.24118253395 & & & & & \\
\hline 1 & 0.999 & 5.23791614199 & & & & & \\
\hline 1 & 1 & 5.23755370619 & 5.23755370619 & 5.23755466744 & & & \\
\hline 1 & 1.001 & 5.49646031081 & & & & & \\
\hline 1 & 1.01 & 5.49452438269 & & & & & \\
\hline 1 & 1.1 & 5.46836520817 & & & & & \\
\hline 1 & 1.2 & 5.42892272878 & & & & & \\
\hline 1 & 1.3 & 5.38170076246 & & & & & \\
\hline
\end{tabular}
agree very well with those obtained by the previous methods (for integer order). This is one of the efficient tests to verify the accuracy of the proposed fractional integration operational matrix of CWs.

Table 4. Comparison of $J^{*}$ for Example 2.
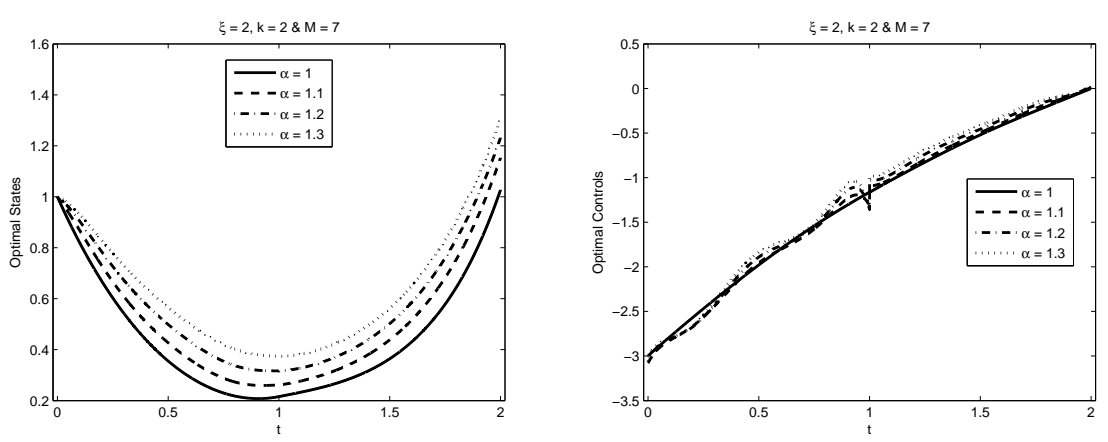

Figure 2. Optimal states and controls, Example 2 with the disturbance. 
Remark 1. By modifying the cost function and the state equation of the system given in Example 2 in Section 5.2 as $J=1 / 2 \int_{0}^{2}\left\{x^{2}(t)+u^{2}(t)\right\} d t$ and $D^{\alpha} x(t)=x(t-1)+u(t)$, we have a problem which has been studied in [17-20]. We solve this problem by making small changes in the model parameters of Example 2 and this is one of the advantages of the method. For all $\alpha$, we have exactly $x^{*}(0)=1$, which is the initial condition of the system and should be satisfied. This indicates that $x^{*}(0)$ is satisfied accurately. A comparison is made in Table 5. The optimal states and controls for some $\alpha$ are shown in Figure 3. The new state equation must be rescaled as $D^{\alpha} x(t)=2^{\alpha} x(t-1 / 2)+2^{\alpha} u(t), 0 \leq t \leq 1$

Table 5. Comparison of $J^{*}$, Remark 1.

\begin{tabular}{llllll}
\hline$\alpha$ & This Work; $k=2, \boldsymbol{M = 7}$ & [20] & [18] & [19] & [17] \\
\hline 1 & 1.647874 & 1.64787419 & 0.4727464 & 0.00002674 & 0.3048 \\
0.99 & 1.648911 & 1.6459912 & 0.4778890 & & \\
0.9 & 1.658451 & 1.6248785 & 0.5021900 & & \\
0.8 & 1.669404 & 1.5926486 & 0.4985242 & & \\
0.7 & 1.680951 & 1.5519859 & & & \\
0.5 & 1.708245 & & & 0.00186172 & \\
\hline
\end{tabular}
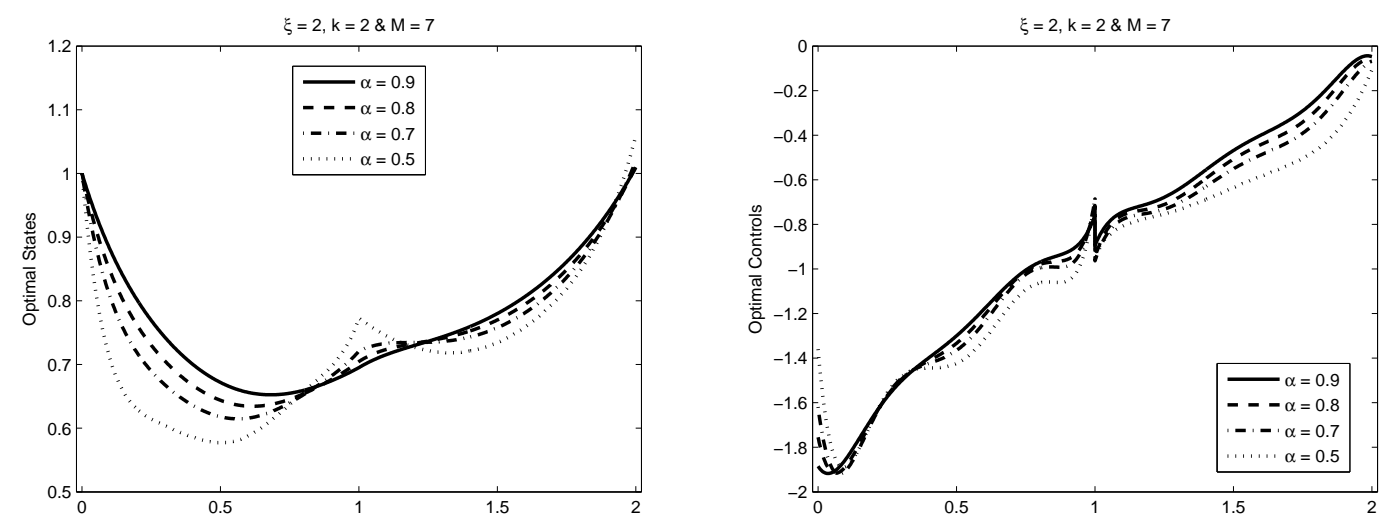

Figure 3. Optimal states and controls, Remark 1.

\subsection{Example 3}

Consider the system [39]

$$
\begin{gathered}
\left\{\begin{array}{l}
D^{\alpha} x_{1}(t)=x_{2}(t) \\
D^{\alpha} x_{2}(t)=-10 x_{1}(t)-5 x_{2}(t)-2 x_{1}(t-1)-x_{2}(t-1)+u(t), \quad 0 \leq t \leq 5
\end{array}\right. \\
\quad \boldsymbol{\theta}(t)=[1,1]^{\top},-1 \leq t<0
\end{gathered}
$$

and the performance index

$$
J=\frac{1}{2} \int_{0}^{5}\left\{10 x_{1}^{2}(t)+x_{2}^{2}(t)+u^{2}(t)\right\} d t
$$

The problem is to find a control $u(t)$ which will steer this system from the initial condition $\mathbf{x}(0)=[1,1]^{\top}$ to the final state conditions $\mathbf{x}(5)=[-1,2]^{\top}$.

In [39], a heavy terminal cost as $J_{t}=10^{6}\left[\left(x_{1}(5)+1\right)^{2}+\left(x_{2}(5)-2\right)^{2}\right]$ is added to the performance index to indicate the terminal constraints. In our method however, by adding the equality constraint to the model of the system we are able to solve such problems, the results are summarized in Table 6 . 
Table 6. Comparison of $J^{*}$ for Example 3.

\begin{tabular}{lll}
\hline$\alpha$ & This Work, $k=2, M=7$ & [39] \\
\hline 1 & 74.1065868949 & 74.1173 \\
0.99 & 75.4717293676 & \\
0.98 & 76.8343875120 & \\
0.97 & 78.1913320078 & \\
0.96 & 79.5404056247 & \\
0.95 & 80.8802694101 & \\
\hline
\end{tabular}

\subsection{Example 4}

Minimize

$$
J=\frac{1}{2} \int_{0}^{1}\left\{\mathbf{x}^{\top}(t)\left[\begin{array}{cc}
1 & t \\
t & t^{2}
\end{array}\right] \mathbf{x}(t)+\left(t^{2}+1\right) u^{2}(t)\right\} d t
$$

subject to

$$
\begin{gathered}
D^{\alpha_{1}} \mathbf{x}(t)-\mathfrak{a}\left[\begin{array}{cc}
-2.7 & 3.1 \\
0 & 0.08
\end{array}\right] D^{\alpha_{2}} \mathbf{x}(t)=\left[\begin{array}{cc}
t^{2}+1 & 1 \\
0 & 2
\end{array}\right] \mathbf{x}\left(t-\frac{1}{2}\right)+\left[\begin{array}{c}
1 \\
t+1
\end{array}\right] u(t)+\left[\begin{array}{c}
t+1 \\
t^{2}+1
\end{array}\right] u\left(t-\frac{1}{4}\right), \\
\begin{cases}\theta(t)=[1,1]^{\top}, & -\frac{1}{2} \leq t \leq 0 \\
\zeta(t)=1, & -\frac{1}{4} \leq t \leq 0,\end{cases}
\end{gathered}
$$

where $\alpha_{1}=1, \alpha_{2}<1$, and $\mathfrak{a}=0,1$.

From the initial state function, we have $\mathbf{x}(0)=\theta(0)$. We solve the problem for different choices of $\alpha_{2}$. The values of $J^{*}$ are given in Table 7. Comparing $J^{*}$ for the case $\mathfrak{a}=0$ with that obtained by our previous research, we see that the accuracy is impressive. In the first choice, where $\mathfrak{a}=1$ and $\alpha_{2}=0.999$, we impose another type of constraint classified as interior point constraints. The interior point constraint is

$$
1.01 x_{1}\left(t_{i}\right)=1.5 x_{2}\left(t_{i}\right)+0.035 \text {, where } t_{i}=t_{f}-h_{v} .
$$

By solving this problem we get $J^{*}=1.51942766043$, Figure 4 shows the optimal states and control in which the value of $\mathbf{x}^{*}\left(t_{i}\right)$ to evaluate the accuracy of the method is given; the interior point constraint is satisfied exactly.


Figure 4. $\mathbf{x}^{*}(t)$ and $u^{*}(t)$ for Case 2 with the interior point constraint, Example 4. 
Table 7. $J^{*}$ for Example 4.

\begin{tabular}{lllll}
\hline $\mathfrak{a}$ & $\boldsymbol{\alpha}_{\mathbf{1}}$ & $\boldsymbol{\alpha}_{\mathbf{2}}$ & This Work & {$[31]$} \\
\hline 0 & 1 & & 1.56224137355 & 1.56224137354 \\
1 & 1 & 0.999 & 1.41013747158 & \\
1 & 1 & 0.99 & 1.41106062244 & \\
1 & 1 & 0.95 & 1.41378641071 & \\
1 & 1 & 0.91 & 1.41668866306 & \\
1 & 1 & 0.9 & 1.41740062973 & \\
1 & 1 & 0.8 & 1.42442691113 & \\
\hline
\end{tabular}

\subsection{Example 5}

Another test is applying the fractional operational matrix directly on the problem possesses an exact solution. We present a simple example to compare the values of the approximated solution by the new fractional integration operational matrix with those obtained by the previous formulation and with the true values of the solution. From Corollary 3 , we do this by setting $k=2$ and selecting the same degree $M=3$ of CWs and, except this operational matrix, all other elements of (49) are the same. We use two operational matrices, as the one is presented in this work and other one was proposed in [30]. Assume that $D^{0.5} x(t)=t^{0.9}$, where $x(0)=1$ and the exact solution is $x(t)=(\Gamma(1.9) / \Gamma(2.4)) t^{1.4}+1$. Here, we apply the fractional operational matrices directly on this problem to integrate $t^{0.9}$ (expanded in its CW series as $\mathbf{U}_{c w}$ ) in the Riemann-Liouville sense. The results of this comparison are given numerically and graphically in Table 8 and Figure 5. We see our fractional integration operational matrix provides more accurate result than the previous presentation. Some statements concerning convergence rate of CW method are presented in [28]. By using the procedure like that mentioned in this reference, we find the convergence is superlinear. From our formulation given in (26)-(29), we get

$\mathbf{P}_{c w}^{0.5}=\left[\begin{array}{cccccc}0.50794909 & 0.2394495 & -0.047889899 & 0.4622839 & -0.12303356 & 0.04291499 \\ -0.079816499 & 0.20317963 & 0.14512831 & 0.092846798 & -0.071331294 & 0.038369003 \\ -0.20113758 & -0.17737905 & 0.17307895 & -0.17585329 & 0.011876661 & 0.011549406 \\ 0 & 0 & 0 & 0.50794909 & 0.2394495 & -0.047889899 \\ 0 & 0 & 0 & -0.079816499 & 0.20317963 & 0.14512831 \\ 0 & 0 & 0 & -0.20113758 & -0.17737905 & 0.17307895\end{array}\right]$.

Table 8. Comparison of errors for Example 5.

\begin{tabular}{lll}
\hline $\boldsymbol{t}$ & This Work; $\xi=\mathbf{2}, \boldsymbol{k}=\mathbf{2 ,} \boldsymbol{M = 3}$ & {$[30] \boldsymbol{k}=\mathbf{2}, \boldsymbol{M}=\mathbf{3}$} \\
\hline 0.1 & 0.00238 & 0.00230 \\
0.3 & 0.00194 & 0.00342 \\
$0.5^{-}$ & 0.00231 & 0.00795 \\
$0.5^{+}$ & 0.00027 & 0.00182 \\
0.7 & 0.00015 & 0.00053 \\
0.9 & 0.00024 & 0.00092 \\
\hline
\end{tabular}


Figure 5. Graphs of solution and error in Example 5. 


\subsection{Example 6}

This problem has been studied in [27]. We want to analyze the fractional delayed damped Mathieu equation as

$$
\ddot{x}(t)+(a+b \cos (\omega t)) x(t)+c D^{\alpha_{1}} x(t)=d D^{\alpha_{2}} x(t-\tau),
$$

where $a=50, b=5, \omega=2, c=1, d=0.5, \tau=1, \theta(t)=\sin t, \tau \leq t \leq 0, t_{f}=10$, and $\dot{x}(0)=1$.

We solve this problem by two techniques:

(1) We use the proposed method directly on this system;

(2) By the technique used in [27], we first select the first derivative of $x(t)$ as a new state which is $x_{2}(t) \triangleq \dot{x}(t)$, and set $x_{1}(t)=x(t)$, then we solve the new problem as

$$
D^{1} \mathbf{x}(t)=\left[\begin{array}{cc}
0 & 1 \\
-50-5 \cos 2 t & 0
\end{array}\right] \mathbf{x}(t)+\left[\begin{array}{cc}
0 & 0 \\
-1 & 0
\end{array}\right] D^{\alpha_{1}} \mathbf{x}(t)+\left[\begin{array}{cc}
0 & 0 \\
0.5 & 0
\end{array}\right] D^{\alpha_{2}} \mathbf{x}(t-1)
$$

where $\theta(t)=[\sin t, \cos t]^{\top}$ is the corresponding initial function. In addition, we consider two cases.

Case 1: $\quad \alpha_{1}=0$ and $\alpha_{2}=0.89$.

Case 2: $\alpha_{1}=0.95$ and $\alpha_{2}=0.85$.

The results of these techniques are shown in Figure 6. There is a good agreement between the two techniques. Using our method, such problems can be solved directly. The method can easily be extended to the case $0<\alpha_{1} \leq 1$, while this may be difficult in some methods like that in [40].
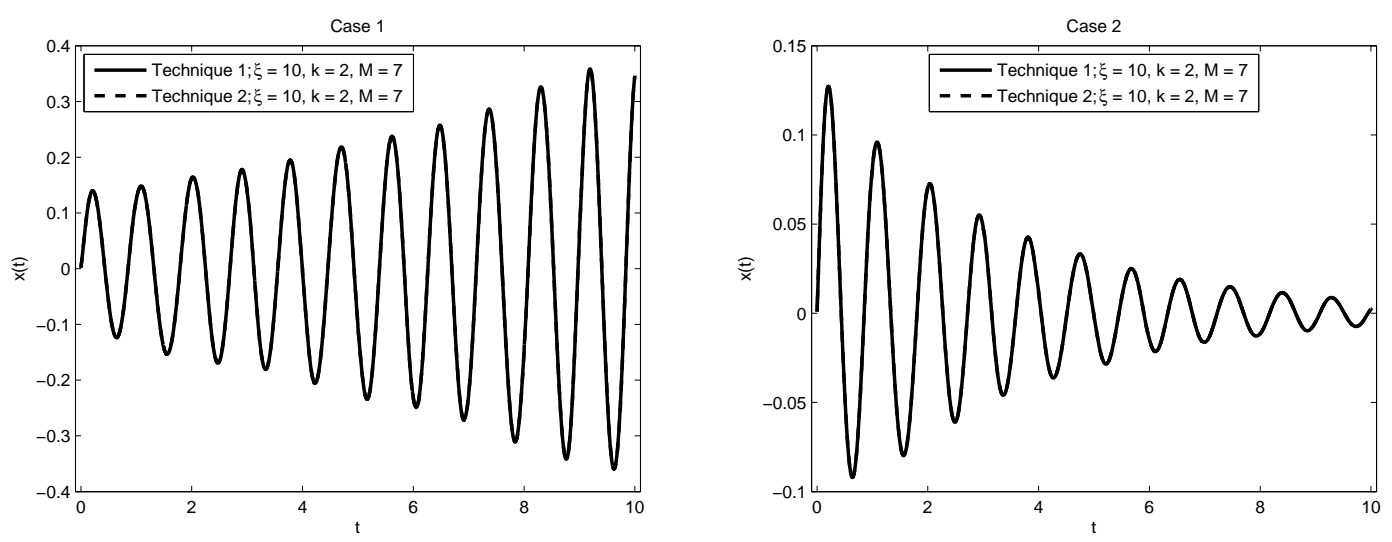

Figure 6. Solutions of Example 6.

\subsection{Example 7}

Finally, we apply the fractional operational matrix on a nonlinear time-delay system, see [41]. Consider the nonlinear fractional time-lag system

$$
\begin{gathered}
D^{\alpha} x(t)=x(t-1) u(t-2), \quad 0 \leq t \leq t_{f} \\
\left\{\begin{array}{l}
\theta(t)=1, \quad-1 \leq t \leq 0 \\
\zeta(t)=0, \quad-2 \leq t \leq 0
\end{array}\right.
\end{gathered}
$$

with the performance index

$$
J=\int_{0}^{t_{f}}\left\{x^{2}(t)+u^{2}(t)\right\} d t
$$

where we have 
Case 1: $t_{f}=3$ and the system is unconstrained.

Case 2: $t_{f}=6$ and the path constraint is $x(t)+u(t) \geq 0.3$.

Case 3: $t_{f}=6$ and the path constraint is $t x(t)+u(t) \geq 0.3$.

We use an approximation scheme in which a nonlinear delay problem is replaced with a sequence of linear delay problems, for an example see [42]. It should be noted that there are some remarkable schemes, like that proposed in [43], that can be implemented by the fmincon function provided by the optimization toolbox in MATLAB. The results of the scheme by using quadprog are reported in Table 9. By comparing $J^{*}$, we see that the method is accurate in this nonlinear problem. For Case 3, the optimal states and controls for some values of $\alpha$ are shown in Figure 7.

Table 9. Comparison of $J^{*}$ for Example 7.

\begin{tabular}{|c|c|c|c|c|c|c|}
\hline \multirow[b]{2}{*}{$\alpha$} & \multicolumn{3}{|l|}{ Case 1} & \multicolumn{2}{|l|}{ Case 2} & \multirow{2}{*}{$\begin{array}{l}\text { Case } 3 \\
\text { This Work }\end{array}$} \\
\hline & This Work & [41] & [44] & This Work & [41] & \\
\hline 1 & 2.761594156 & 2.761599 & 2.761837 & 3.108192976 & 3.108259352 & 3.764357269 \\
\hline 0.99 & 2.758815336 & & & 3.111200334 & & 3.766855849 \\
\hline 0.9 & 2.733594641 & & & 3.154392881 & & 3.801712657 \\
\hline
\end{tabular}
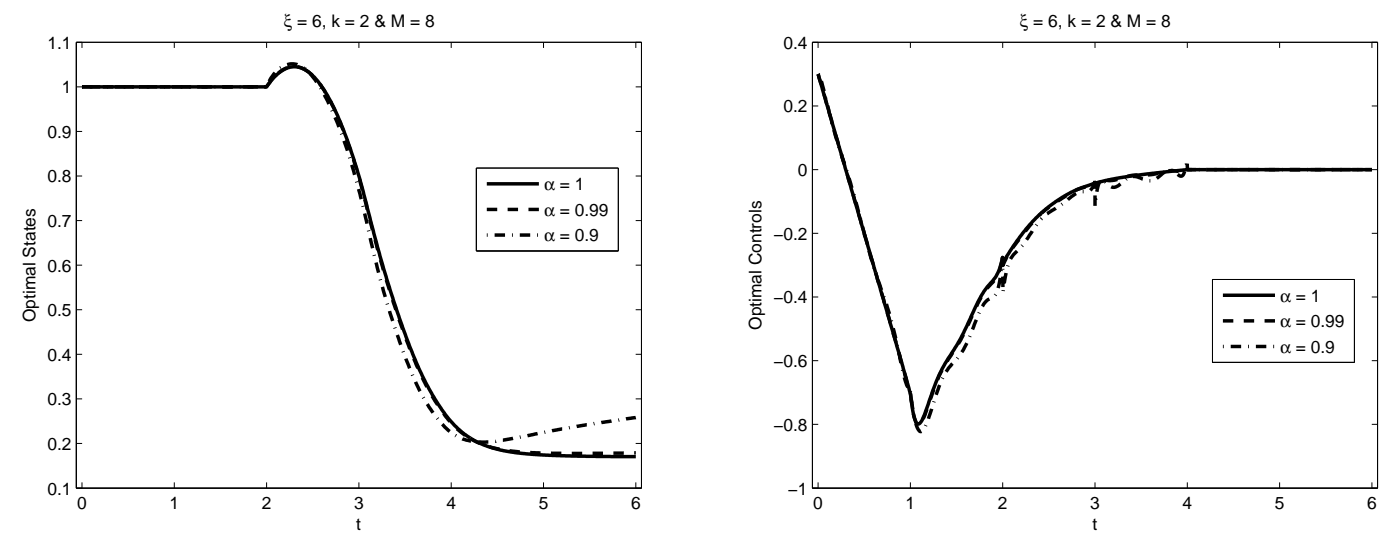

Figure 7. Optimal states and controls for Example 7, Case 3.

\section{Conclusions}

Fractional integration operational matrix of Chebyshev wavelets based on Riemann-Liouville sense has been obtained. With the use of this matrix, a simple method for optimal control of fractional linear-quadratic time delay systems and also a framework for the analysis of multifractional time-delay systems have been presented. We have seen that the new formulation is accurate and can be applied on the cases we have $\alpha=1$. The proposed method can be applied to multifractional optimal control problems and also to more complicated situations in which systems are subject to some constraints. In future work, we can use the presented concepts to the optimal control of nonlinear fractional systems.

Funding: This research received no external funding.

Acknowledgments: The author would like to thank the Editor and Reviewers for their useful suggestions to improve the quality of the paper.

Conflicts of Interest: The author declares no conflict of interest. 


\section{References}

1. Miller, K.S.; Ross, B. An Ontroduction to the Fractional Calculus and Fractional Sifferential Equations; John Wiley \& Sons: Hoboken, NJ, USA, 1993.

2. Podlubny, I. A Fractional Differential Equations: An Introduction to Fractional Derivatives, Fractional Differential Equations, to Methods of Their Solution and Some of Their Applications; Academic Press: New York, NY, USA, 1999.

3. Sabatier, J., Agrawal, O.P., Tenreiro Machado, J.A. (Eds.) Advances in Fractional Calculus, Theoretical Developments and Applications in Physics and Engineering; Springer: Berlin/Heidelberg, Germany, 2007.

4. Nutting, P.G. A New General Law of Deformation. J. Frankl. Inst. 1921, 191, 679-685. [CrossRef]

5. Bagley, R.L.; Torvik, P.J. Fractional Calculus, a Different Approach to the Analysis of Viscoelastically Damped Structures. AIAA J. 1983, 21, 741-748. [CrossRef]

6. Suarez, L.E.; Shokooh, A. An Eigenvector Expansion Method for the Solution of Motion Containing Fractional Derivatives. J. Appl. Mech. 1997, 64, 629-635. [CrossRef]

7. Atanacković, T.M.; Pilipović, S.; Stanković, B.; Zorica, D. Fractional Calculus with Applications in Mechanics; John Wiley \& Sons: Hoboken, NJ, USA, 2014.

8. Bohannan, G.W. Analog fractional order controller in temperature and motor control applications. J. Vib. Contr. 2008, 14, 1487-1498. [CrossRef]

9. Akgül, A.; Kılıçman A. Improved $\left(G^{\prime} / G\right)$-Expansion Method for the Space and Time Fractional Foam Drainage and KdV Equations. Abst. Appl. Anal. 2013, 2013, 414353. [CrossRef]

10. Inc, M.; Akgül, A. Approximate solutions for MHD squeezing fluid flow by a novel method. Bound. Value Probl. 2014, 2014, 18. [CrossRef]

11. Ragab, A.A.; Hemida, K.M.; Mohamed, M.S.; Abd El Salam, M.A. Solution of Time-Fractional Navier-Stokes Equation by Using Homotopy Analysis Method. Gen. Math. Notes 2012, 13, 13-21.

12. Edeki, S.O.; Akinlabi, G.O. Coupled Method for Solving Time-Fractional Navier-Stokes Equation. Int. J. Circuits Syst. Signal Process. 2018, 12, 27-34.

13. Sahu, P.K.; Saha Ray, S. Comparison on wavelets techniques for solving fractional optimal control problems. J. Vib. Contr. 2018, 24, 1185-1201. [CrossRef]

14. Birs, I.; Muresan, C.; Nascu, I.; Ionescur, C. A Survey of Recent Advances in Fractional Order Control for Time Delay Systems. IEEE Access 2019, 7, 30951-30965. [CrossRef]

15. Johnson, M.A.; Moon, F.C. Experimental characterization of quasiperiodicity and chaos in a mechanical system with delay. Int. J. Bifurc. Chaos 1999 9, 49-65. [CrossRef]

16. Dabiri, A.; Nazari, M.; Butcher, E.A. Optimal fractional state feedback control for linear fractional periodic time-delayed systems. In Proceedings of the American Control Conference (ACC), Boston, MA, USA, 6-8 July 2016; pp. 2778-2783. [CrossRef]

17. Rahimkhani, P.; Ordokhani, Y.; Babolian, E. An efficient approximate method for solving delay fractional optimal control problems. Nonlinear Dyn. 2016, 86, 1649-1661. [CrossRef]

18. Bhrawy, A.H.; Ezz-Eldien, S.S. A new Legendre operational technique for delay fractional optimal control problems. Calcolo 2016, 53, 521-543. [CrossRef]

19. Rabiei, K.; Ordokhani, Y.; Babolian, E. Fractional order Boubaker functions and their applications in solving delay fractional optimal control problems. J. Vib. Contr. 2017, 1-14. [CrossRef]

20. Moradi, L.; Mohammadi, F.; Baleanu, D. A direct numerical solution of time-delay fractional optimal control problems by using Chelyshkov wavelets. J. Vib. Contr. 2018, 1-15. [CrossRef]

21. Lakshmikantham, V. Theory of fractional functional differential equations. Nonlinear Anal. 2008, 69, 3337-3343. [CrossRef]

22. Daftardar-Gejji, V.; Sukale, Y.; Bhalekar, S. Solving fractional delay differential equations: A new approach. Fract. Calc. Appl. Anal. 2015, 18, 400-418,

23. Saedshoar Heris, M.; Javidi, M. On fractional backward differential formulas methods for fractional differential equations with delay. Int. J. Appl. Comput. Math. 2018, 4. [CrossRef]

24. Maleki, M.; Davari, A. Fractional retarded differential equations and their numerical solution via a multistep collocation method. Appl. Numer. Math. 2019, 143, 203-222. [CrossRef]

25. Dabiri, A.; Butcher, E.A. Efficient modified Chebyshev differentiation matrices for fractional differential equations. Commun. Nonlinear Sci. Numer. Simul. 2017, 50, 284-310. [CrossRef] 
26. Parsa Moghaddam, B.; Salamat Mostaghim, Z. Modified Finite Difference Method for Solving Fractional Delay Differential Equations. Boletim da Sociedade Paranaense de Matemática 2017, 35, 49-58. [CrossRef]

27. Dabiri, A.; Butcher, E.A. Numerical Solution of Multi-Order Fractional Differential Equations with Multiple Delays via Spectral Collocation Methods. Appl. Math. Model. 2017, 56, 424-448, doi:10.1016/j.apm.2017.12.012. [CrossRef]

28. Malmir, I. Novel Chebyshev wavelets algorithms for optimal control and analysis of general linear delay models. Appl. Math. Model. 2019, 69, 621-647. [CrossRef]

29. Li, Y. Solving a nonlinear fractional differential equation using Chebyshev wavelets. Commun. Nonlinear Sci. Numer. Simul. 2010, 15, 2284-2292. [CrossRef]

30. Heydari, M.H.; Hooshmandasl, M.R.; Cattani, C. A new operational matrix of fractional order integration for the Chebyshev wavelets and its application for nonlinear fractional Van der Pol oscillator equation. Proc. Indian Acad. Sci. (Math. Sci.) 2018, 128, 1-26. [CrossRef]

31. Malmir, I. Optimal control of linear time-varying systems with state and input delays by Chebyshev wavelets. Stat. Optim. Inf. Comput. 2017, 5, 302-324. [CrossRef]

32. Abramowitz, M., Stegun, I.A. (Eds.) Handbook of Mathematical Functions with Formulas, Graphs, and Mathematical Tables; Dover Publications: New York, NY, USA, 1972.

33. Malmir, I. A novel wavelet-based optimal linear quadratic tracker for time-varying systems with multiple delays. arXiv 2018, arXiv:1802.05618.

34. Malmir, I. Legendre wavelets with scaling in time-delay systems. Stat. Optim. Inf. Comput. 2019, 7, $235-253$. [CrossRef]

35. Basin, M.; Rodriguez-Gonzalez, J.; Fridman, L. Optimal and robust control for linear state-delay systems. J. Frankl. Instit. 2007, 344, 830-845. [CrossRef]

36. Currie, J.; Wilson, D.I. OPTI: Lowering the Barrier Between Open Source Optimizers and the Industrial MATLAB User. In Proceedings of the Foundations of Computer-Aided Process Operations, Savannah, GA, USA, 8-13 January 2012.

37. Luus, R. Iterative Dynamic Programming Monographs and Surveys in Pure and Applied Mathematics; Chapman \& Hall/CRC: Boca Raton, FL, USA, 2000.

38. Smith, S.F. Optimal control of delay differential equations using evolutionary algorithms. Complex. Int. 2005, 12, 1-10.

39. Chan, H.C.; Perkins, W.R. Optimization of time delay systems using parameter imbedding. Automatica 1973, 9, 257-261. [CrossRef]

40. Kumar, P.; Agrawal, O.P. Numerical Scheme for the Solution of Fractional Differential Equations of Order Greater Than One. J. Comput. Nonlinear Dyn. 2005, 1, 178-185. [CrossRef]

41. Göllmann, L.; Kern, D.; Maurer, H. Optimal control problems with delays in state and control variables subject to mixed control-state constraints. Optim. Cont. Appl. Meth. 2009, 30, 341-365. [CrossRef]

42. Banks, H.T. Approximation of Nonlinear Functional Differential Equation Control Systems. J. Optim. Theory Appl. 1979, 29, 383-408. [CrossRef]

43. Bouafoura, M.K.; Braiek, N.B. Hybrid Functions Direct Approach and State Feedback Optimal Solutions for a Class of Nonlinear Polynomial Time Delay Systems. Complexity 2019, 2019, 9596253. [CrossRef]

44. Hosseinpour, S.; Nazemi, A. A collocation method via block-pulse functions for solving delay fractional optimal control problems. IMA J. Math. Cont. Inf. 2016, 34, 1215-1237. [CrossRef]

(c) 2019 by the author. Licensee MDPI, Basel, Switzerland. This article is an open access article distributed under the terms and conditions of the Creative Commons Attribution (CC BY) license (http://creativecommons.org/licenses/by/4.0/). 